\title{
Factors affecting Acheulean handaxe variation: Experimental insights, microevolutionary processes, and macroevolutionary outcomes
}

Stephen J. Lycett ${ }^{1 *}$, Kerstin Schillinger ${ }^{1}$, Metin I. Eren ${ }^{2,3}$, Noreen von Cramon-Taubadel ${ }^{1}$, Alex Mesoudi ${ }^{4}$

${ }^{1}$ Department of Anthropology, University at Buffalo SUNY, 380 MFAC-Ellicott Complex, Buffalo, NY 14261, U.S.A.

${ }^{2}$ Department of Anthropology, University of Missouri, Columbia, MO 65211, U.S.A.

${ }^{3}$ Department of Archaeology, Cleveland Museum of Natural History, Cleveland, OH, 44106, U.S.A.

${ }^{4}$ Department of Biosciences, College of Life and Environmental Sciences, University of Exeter, Penryn Campus, Penryn, TR10 9FE, U.K.

*Correspondence to: sjlycett@,buffalo.edu

Key words: Acheulean, handaxes, social learning, raw material, imitation, emulation, heritability

\begin{abstract}
The so-called "Acheulean" is comprised of individual knapping events undertaken by individual hominins. In other words, it is a particular component of hominin behavior that we draw out and amalgamate into a wider "pattern." The resultant phenomenon (i.e., "the Acheulean") is an entity that stretches over the space of three continents and spans a time period in excess of one million years. If such an exercise has any merit, it is because it provides a means of comparative (behavioral) analysis over these swathes of time and space. Comparative research can document, measure, and statistically assess temporo-spatial patterns of artifactual variation, and so test hypotheses regarding the character of that variation. However, comparative research alone is not sufficient to fully understand this archaeological legacy. Here, we review and synthesize recent experimental work that we have undertaken, which has specifically investigated some of the factors potentially responsible for the generation and constraining of variation within the Acheulean techno-complex. We examine issues of raw material, copying errors, and their relationship to mechanisms of social learning. Understanding these microevolutionary factors via experiments, we contend, is essential in order to reach a secure understanding of the macroscale phenomenon typically referred to as the "Acheulean."
\end{abstract}




\section{Introduction}

The first one and a half million (plus) years of knapped stone tool technology consisted of cores and the sharp flakes struck from them (Semaw, 2000; Roche, 2005; Schick and Toth, 2006; Harmand et al., in press). Despite their seeming simplicity, the routine production of such cutting tools appears unique to the hominin lineage (Roux and Bril, 2005; Shumaker et al., 2011), and consequently, this technological innovation is regarded as a fundamental step along the journey toward the eventual emergence of our own species (Isaac, 1983; Shipman and Walker, 1989; Ambrose, 2001; Rogers and Semaw, 2006). From around 1.7-1.5 million years ago, however, hominins began to produce entirely new forms of artifacts, most notably, so-called "handaxes" (Lepre et al., 2011; Beyene et al., 2013). The production of these novel artifacts marked a shift away from cores and nodules being seen simply as an item to be struck in order to produce flakes, to a situation where knapping events were strung together in a manner that resulted in a characteristic residual form-i.e., the "handaxe" (Roche, 2005; Gowlett, 2006). Although much remains to be learned regarding details of their functions and applications in specific circumstances, in general terms, the archaeological contexts of such artifacts, residue analyses, cut-mark analyses, design theory, and experiments, combine to suggest they plausibly performed a variety of functions as cutting and/or chopping tools (Jones, 1980; Roberts and Partfitt, 1999; Domínguez-Rodrigo et al., 2001; Simão, 2002; Gowlett, 2006; Bello et al., 2009; Yravedra et al., 2010; Solodenko et al., 2015).

Following their initial appearance, the production of handaxes was an activity undertaken by hominin populations for over one million years (Haslam et al., 2011). Moreover, hominins engaged in production of these tools over a geographic range that stretched from South Africa to Britain, and from the Iberian Peninsula to the Indian subcontinent (Clark, 1994; Schick, 1998; Gowlett, 2011). Indeed, it is now clear that definite pockets of handaxe production took place east of the so-called "Movius Line," even if debates regarding the chronology, comparability, and relationship of these East Asian examples rumble on (Hou et al., 2000; Norton et al., 2006; Lycett and Gowlett, 2008; Petraglia and Shipton, 2009; Norton and Bae, 2009; Lycett and Bae, 2010; Li et al., 2014; Wang et al., 2012, 2014). Rightly or wrongly, the geographic and temporal expanse of these characteristic artifacts has famously been referred to in the collective as "the Acheulean" (Wynn and Tierson, 1990; Schick, 1998; Gowlett, 2011). As recent commentators have noted, although this entity has curiously been defined in a variety of different ways over the years, it is the presence of handaxes more than any other criterion that tends to lead to a particular site or assemblage being characterized as 
"Acheulean" (Lycett and Gowlett, 2008; Diez-Martín and Eren, 2012; Domínguez-Rodrigo et al., 2014).

By any standards, the "Acheulean" is a phenomenon that evokes immediate questions. In basic terms, it simply represents a pattern of repeated hominin behavior (i.e., biface production) over the course of one million (plus) years over three different continents. This repetition of handaxe production is, of course, likely driven by shared functional needs on the part of its producers over time and space; as Gowlett (2011: 100) has put it, "a culturally maintained set of functional solutions to everyday tasks which recur." However, that commonality of behavioral pattern —at least in general termsprovides a basis for comparative research at the archaeological level (Wynn and Tierson, 1990; Vaughan, 2001; Lycett and Gowlett, 2008; Petraglia and Shipton, 2009; Chauhan, 2010; Wang et al., 2012). In other words, an essential part of studying the Acheulean is to understand its potential variability, both in temporal and geographical terms. Comparative research can document, measure, and statistically assess temporo-spatial patterns of artifactual variation, and so test hypotheses regarding the character of that variation. However, experiments, we contend, are an additional but vital way in which the mechanisms that both generate and constrain variation within the Acheulean can be more securely understood. Here, we review and synthesize recent experimental work that we have undertaken, which has specifically investigated some of the factors potentially responsible for the generation and constraining of variation within the Acheulean techno-complex. We relate our discussion of this experimental work to cultural microevolutionary considerations, which specifically relates patterns of variation to issues of social learning. In closing, we show how an understanding of these issues is an essential element in understanding Acheulean temporo-spatial variation, and the constraint of that variation, in cultural evolutionary terms. In other words, this synthesis of various experimental findings and microevolutionary principles provides insights regarding the Acheulean at the macroevolutionary level.

\section{Acheulean variation in the raw: toolstone "constraints" investigated experimentally}

Raw material factors have long been considered to exert an influence on the form and composition of lithic assemblages (Goodman, 1944), and the role of raw material has been frequently deliberated over in specific regard to variation within the Acheulean techno-complex (e.g., Isaac, 1977; Jones, 1979; Wynn and Tierson, 1990; Roe, 1994; Schick 1994; Clark, 2001; Noll and Petraglia, 2001; Sharon, 2008; Archer and Braun, 2010; Costa, 2010; Wang et al., 2012; among many others). The 
suspected link between lithological factors and resultant artifactual form is, of course, a logical outcome of the fact that the medium with which any artisan is working might have properties that affect given outcomes. Both the internal and external properties of rock types have been considered within the context of such debates. In the case of a rock's internal characteristics, factors such as isotropy, homogeneity, brittleness, hardness, and granularity have frequently been considered pertinent, all of which ultimately relate to the mineralogy and microstructure of particular rock types (Goodman, 1944; Callahan, 1979; Whittaker, 1994; Andrefsky, 1998). External characteristics that may be relevant include the size, shape, and regularity of the material to be knapped, as well as whether cortex is present or absent (Ashton and McNabb, 1994; Jennings et al., 2010; Smallwood, 2010; Eren et al., 2011). The presence of cortex is potentially important since it has been shown that rock types possessing cortex may in fact behave in terms of some properties (specifically rebound hardness) in two distinct ways, with cortical material acting as one distinct rock type, while the uncortical surface of the same rock can act more similarly to other rock types entirely (Eren et al., 2014).

The seemingly logical notion that rock variability will be a major, if not the major factor, driving variability in Acheulean handaxe form could, however, be potentially overstated. Artifacts, by definition, involve a behavioral component in their formation. There is obviously a risk of circularity in assuming that just because rock types differ at two different Acheulean localities and the properties of artifacts at those two sites also differ, then causality for the latter must automatically reside in the former. Indeed, based on empirical study of Acheulean assemblages, some have recently questioned the extent to which variability in the forms of artifacts such as handaxes is necessarily driven solely by raw material factors (e.g., Sharon, 2008; Costa, 2010).

As we have noted, however, handaxes are the product of multiple knapping events sequentially strung together in order to produce their characteristic properties (Roche, 2005; Gowlett, 2006). If raw material exerts an influence at each individual flaking event, then such effects will obviously be cumulative, potentially leading to divergent outcomes in differing rock types, even if a knapper is striving toward the same overall goal. A major point to consider here, therefore, is whether differences in the internal and external properties of different rock types automatically conspire to produce statistical differences in handaxe form in such a manner. Addressing this specific question on the basis of the archaeological record alone poses serious challenges, not least of which is that 
directly relevant factors - such as differing knapper skills and intentions-cannot be observed directly and might also vary from site to site. Experimental approaches to this issue, however, have the advantage that factors such as knapper skill and intention can be strategically controlled (i.e., held constant) and so help highlight any particular effect that raw material may exert in this context.

Given these factors, we (Eren et al., 2014) recently undertook an experiment designed expressly to determine if raw material differences automatically result in divergences in the statistical shape properties of handaxes produced on them. One of us (MIE), an expert knapper with 13 years of experience at the time of the experiment, undertook the task of copying a specific "target" handaxe from nodules of three distinct rock types: flint, basalt, and obsidian. Prior to the experiment, a series of formal analyses were undertaken to assess differences in the internal and external properties of these three categories of raw material. Measurements $(n=7)$ taken on the external dimensions each of the nodules $(\mathrm{n}=105)$ were subjected to statistical analysis. These analyses indicated that both the initial sizes and shapes (size-adjusted data) of nodules in each raw material class exhibited statistically significant differences. Thin section microscopy, rebound hardness tests, and biaxial flexure analyses were undertaken to examine the microstructure, mineralogy, and mechanical properties of each raw material. These tests indicated distinct differences in the mineralogy, hardness (rebound) values, and mechanical behavior (peak breakage loads) across each of the three raw materials (see Eren et al., 2014 for full results).

The expert knapper (MIE) was instructed to copy the shape of a handaxe made by another of the investigators (SJL). The target "model" handaxe (Figure 1) was manufactured on flint from a different source from that used in the experimental data, and exhibited an overall roughly symmetrical profile in three dimensions with low width/thickness ratio (3.33:1), so resembling handaxes of the "Late Acheulean" (Edwards, 2001; Wynn, 2002). This form of handaxe was deliberately chosen for the experiment so as to challenge the knapper within the context of Acheulean handaxe variation as a whole. The knapper then attempted to copy the shape of the model handaxe using 35 nodules of each of the three different raw materials, thus producing a total of 105 experimental handaxes. To mitigate the effects of learning during the course of knapping the replicas, the order in which different materials were knapped was rotated (flint, basalt, and obsidian). In knapping the replicas, the same set of tools was used throughout the experiment (three antler billets and four hammer stones). 
Following this stage, a dataset of 29 morphometric variables was obtained for each replica handaxe and the target-model using a standardized orientation protocol (Schillinger et al., 2014a). Thereafter, Eren et al. (2014) transformed the obtained linear measurements into shape variables via the geometric mean method (Jungers et al., 1995; Lycett et al., 2006). This method efficiently controls for scaling (i.e., size) variation between objects by creating a dimensionless, scale-free variable while preserving shape variation between them (Falsetti et al., 1993; Jungers et al., 1995). For the main analyses, we predicted that if raw material properties (external and/or internal) were an absolute driver of handaxe shape, then statistically significant differences in shape should be determinable across the handaxes made on each category of raw material. Moreover, it might also be reasonably predicted that if raw material differences were systematically influencing the knapper's ability to copy the shape of the model accurately, then the magnitude of copying errors in specific handaxe raw material groups should be statistically different. These predictions were tested using a combination of univariate and multivariate statistical methods.

A multivariate analysis of variance (MANOVA) of the 29 size-adjusted shape variables indicated no significant differences between the three groups of handaxes made on each of the different raw material classes using two different test statistics (Wilks' Lambda: $F=0.893$, df $=148, p=0.684$; Pillai's Trace: $F=0.902, \mathrm{df}=150, p=0.668$ ). In other words, handaxe shapes were not significantly different across the three different categories of raw material (Figure 2). Indeed, overall levels of shape variability in each of the three raw material categories and their comparability to the targetmodel handaxe can be visualized using Principal Component Analysis (PCA). Figure 3 shows the output from this PCA analysis. As can be seen, consistent with the MANOVA results, the model falls close to the center of the variability seen in the handaxes made on all three raw materials. We also undertook an analysis of variance (ANOVA) on the multivariate Euclidean distances between each replica and the model using all the available PC scores. This enabled us to test for significant differences in the average divergence of shape between the replica handaxes and the model across the different raw material groups. The ANOVA found no significant differences $(F=1.886 ; p=0.157)$, indicating that the overall magnitude of copying errors across handaxe raw material groups was not statistically different. 
In sum, Eren et al.'s (2014) analyses indicate that the demonstrable differences in the external and internal properties of the three raw material categories were not systematically influencing the knapper's ability to copy the shape of the target-model handaxe. Indeed, we even found that in terms of central tendencies, the economy of reduction was statistically identical across raw materials, thus indicating that — on average - the knapper did not need to remove significantly different quantities of material to achieve the same shape effects in the three different raw materials. At this juncture it should be emphasized that these results do not indicate that raw material is of no influence when examining Acheulean handaxe variation in the archaeological record. What they do demonstrate, however, is that evident differences in raw materials do not automatically or necessarily result in differences of handaxe shape. Thus, these results demonstrate the dangers of assuming that raw material factors are the primary driver of between-assemblage handaxe variation in the archaeological record. In other words, they emphasize an inherent problem in assuming that just because handaxe shape properties at different Acheulean localities differ, and raw materials at those same sites also differ, that causality for the shape differences can be automatically attributed to raw material factors.

These results further demonstrate the potential for knapper behavior to mitigate differences in the internal and external properties of various raw materials, and so ameliorate their effects at the level of the eventual artifact. Indeed, the results of this experiment are congruent with several studies of archaeological data, which suggest that to fully explain differences in the attributes of stone artifacts across time and space, factors other than raw materials must be invoked (e.g., Sharon, 2008; Clarkson, 2010; Costa, 2010; Smallwood, 2012; Buchanan et al., 2014). In fact, although standard logic has frequently suggested otherwise, on the basis of the results described, it may be more appropriate to rule out other factors prior to attributing differences in lithic assemblages to raw material effects, just as in comparative biology, where internal historical factors are controlled for prior to asserting a prominent role for external factors (e.g., Nunn, 2011). Indeed, we are by no means the first to urge controlling for cultural-historical dynamics prior to asserting a role for extraneous causation in behavioral data (e.g., Mace and Pagel, 1997).

\section{Social learning and Acheulean variation: mechanisms and mutation}

A specific aim of cultural evolutionary approaches is to study the mechanisms and processes that underlie particular temporal and spatial trends (e.g., Cavalli-Sforza and Feldman, 1981; Boyd and 
Richerson, 1985; O’Brien and Lyman, 2000; Mesoudi and O'Brien, 2008; Mesoudi, 2011; Shennan, 2011; Jordan, 2015; Lycett, 2015). Specifically, this framework emphasizes the co-occurrence of three properties that operate in forming a system of "descent with modification" (i.e., cultural evolution). These three properties are: (1) a mechanism of social learning that acts as a mode of inheritance; (2) that entities within a population vary in their characteristics; and (3), that various factors (random and/or non-random) create a situation whereby variation (discrete or continuous) seen in one generation is not necessarily represented in identical frequency in the following generation (Mesoudi, et al., 2004). Inevitably, therefore, this framework simultaneously emphasizes the study of mechanisms that allow fidelity (i.e., continuity) in the case of traditions, while also asking questions regarding the generation of variation.

As we have noted, one of the most striking features of the Acheulean is its endurance over time and space as a behavioral pattern: repeated bouts of handaxe production over three continents and for in excess of one million years. This durability is perhaps even more striking in the light of recent work that has highlighted how traditions of handaxe manufacture are likely to have been inherently unstable due to key microevolutionary processes operating at the level of the individual knapper. For instance, Kempe et al. (2012) recently examined the effect of copying error on size variation in Acheulean handaxes, a phenomenon that is an inevitable feature whenever artifact forms are copied manually (Eerkens, 2000; Eerkens and Lipo, 2005; Hamilton and Buchanan; 2009; Schillinger et al., 2014a, 2014b). Kempe et al. (2012) simulated the effects of cumulative copy errors in perceptions of an artifact's size utilizing average error rates (3.43\% for a given dimension), which they had established via experiments using images of Acheulean handaxes. Their experimentally-informed simulations suggested that in as little as 4,000 years, variation resulting from cumulative copy errors would exceed that seen in a large sample $(n=2061)$ of temporally diverse Acheulean handaxes. Kempe et al. (2012) concluded that specific processes must, therefore, have counteracted the inevitable increase in variation generated by perceptual copying error in relation to size parameters of Acheulean handaxes.

It is important to note that Kempe et al.'s (2012) experiment examined only error that might accrue from copying the size (i.e., scale) of dimensional attributes associated with Acheulean handaxes. However, as we have noted, it is the characteristic shape properties of handaxes that are perhaps their most prominent feature (Wynn, 1995; Roche, 2005). Size and shape are two distinct properties of any 
physical object (Bookstein, 1989; Jungers et al., 1995). Put simply, size is a univariate property of any artifact and, as such, can be adequately described by a single continuous variable such as "volume" or, in one dimension, by a single attribute such as "length." Shape, however, is intrinsically a multivariate property, which cannot be adequately described univariately, depending as it does on the necessary interrelationship between multiple aspects of an artifact's form. Handaxes, by definition, have specific three-dimensional shape properties over their surface areas, especially relating to the interrelationship between multiple dimensions of their relative length, width, and thickness attributes (Gowlett, 2006).

Schillinger et al. (2014a) recently demonstrated that the shape properties of knapped artifacts, such as handaxes, are likely to be particularly prone to copy errors, even on a relative basis with other classes of artifact. This experiment specifically tested a hypothesis originally put forward by Deetz (1967), which proposed that artifacts produced via "reductive" manufacturing techniques would be more prone to copying errors than those produced via manufacturing processes that can incorporate both the addition and removal of material. Knapping, of course, is an inherently reductive manufacturing process; no flake, once removed can be added back onto the core to correct an error (Baumler, 1995: 11). The manufacture of an artifact such as a pot, however, is not so limited; material can either be added or removed to create the final shape. According to Deetz (1967), the implications of this are that different manufacturing processes may have inherently different tendencies in terms of artifactual copying error.

Schillinger et al. (2014a) tested this hypothesis experimentally, with specific regard for shape copying error independently of size. Experimental participants took part in an activity which involved copying the shape of a "target" artifact (a replica handaxe made on flint) from a standardized block of modelling clay using a steel table knife. The participants were divided into two different experimental conditions. In the first condition, participants $(n=30)$ were instructed to copy the "target" model by only removing material, whereas participants in the alternative condition $(\mathrm{n}=$ 30) were instructed that they could both freely remove and add material during the course of the experiment. Mean shape copy error rates (determined by morphometric analysis) in the two conditions were then compared statistically. A conservative nonparametric test clearly established that mean shape copying error rates were significantly higher (Mann-Whitney $U=621.5$, Monte Carlo $p=0.0199$ ) in the "reductive-only" condition compared to the "additive-reductive" condition. 
In other words, Schillinger et al.'s (2014a) experiment established that "mutation rates" in the shape properties of artifacts resulting from copy error are process dependent, and that reductive processes are especially error prone.

Such findings are particularly relevant in considerations of Acheulean handaxe variation, since they suggest that such artifacts - necessarily produced via a reductive process of knapping-will have relatively high shape mutation rates, at least on a comparative basis with artifacts produced via alternative means. Taking Kempe et al.'s (2012) and Schillinger et al.'s (2014) results together, this would imply that the size and shape characteristics of handaxes observed in the Acheulean will have been inherently unstable, even if learning of handaxe properties was guided to some extent by copying the form of artifacts made by others. The potency of shape mutation rates instigated by this type of process is amply illustrated in Figure 4, based on previously unpublished data. Here, 15 experimental participants were asked to copy the three-dimensional shape properties of an artifact in the form of a "transmission chain," whereby each participant copied the "artifact" produced by a previous participant. Transmission chains of this form are being increasingly used to study social learning processes (Mesoudi and Whiten, 2008). The transmission chain in Figure 4 was initiated by use of a "target" model artifact, which was deliberately designed to resemble an Acheulean handaxe. The "target" model and all subsequent copies of it were manufactured from standardized blocks of foam $(22.3 \times 11 \times 7.8 \mathrm{~cm})$ using a plastic table knife. The high-density foam blocks used in this experiment are designed to be used by professional florists to securely hold the stems of artificial flowers. The material is robust to handling, but designed to be malleable so that it can be easily cut and modified into desired sizes and shapes using commonplace implements such as knives and scissors. All participants were instructed to copy the shape of the previous participant's artifact as accurately as possible, whom they had not seen manufacture the piece (i.e., they saw only the end product of the previous stage in the chain). The rapid degradation of shape that occurs in both planform (Figure 4a) and profile views (Figure 4b) can readily be seen over the course of the 15 transmission events. Although just a single example, this transmission chain visually illustrates the potentially large effects of cumulative copying error, which could result in the degradation and eventual "collapse" of a particular behavioral pattern (e.g., handaxe manufacture).

Given these findings regarding the inherent instability of any reductive artifactual tradition that contains specific shape properties, the temporal and spatial entity referred to as "the Acheulean" is 
arguably more striking than ever. Put simply, it is reasonable to ask what mechanisms are likely to have been in place to countermand such mutation rates. Some (e.g., Mithen, 1999) have suggested that the spatio-temporal duration of the Acheulean alone must imply the existence of an imitative learning mechanism, whereby not merely the artifact form was copied (emulative learning), but that aspects of techniques and behavioral gestures used by hominins involved in handaxe manufacture must also have been copied (imitative learning). Individual ("trial-and-error") learning might also have helped to reduce the effects of shape copying error in traditions of handaxe manufacture, at least once the notion of a "handaxe" had been instilled via other mechanisms of social learning such as stimulus enhancement and emulation (for definitions of these various social learning mechanisms, see e.g., Whiten et al., 2004; Lycett, 2015).

As recently noted by Lycett et al. (2015), however, there are reasons to be cautious that trial-anderror learning alone can explain the scale of the Acheulean in the face of relevant mutation rates. One pertinent factor here, which has gone surprisingly under-discussed in considerations of Acheulean social learning is the inherent costs and risks involved in knapping. Knapping obviously involves the deliberate removal of razor-sharp flakes via percussive striking of a stone mass, as well as smaller sharp chips being thrown into the air. In such an environment, painful open wounds, bleeding, eye damage/loss, risk of infection to wounds, as well as sprains or damage to ligaments and muscles, are all occupational hazards. Indeed, the ethnographic literature relating to historically documented peoples that rely (or relied) upon knapped stone technology makes reference to such injury risks (e.g., Pope, 1918: 117; Kroeber, 1961: 184; Hampton, 1999: 267). Contemporary knappers that engage in this craft for scholarly reasons and/or recreation wear protective equipment such as eyegoggles, work gloves, leather pads, and leather hand-scraps in a direct attempt to avoid these kinds of injury. One contemporary flintknapper severed the tendons in a finger with a seemingly innocuouslysized flake $(\sim 20 \times 5 \mathrm{~mm})$ and which, even despite corrective surgery, led to permanent debilitation in the knapper's hand (Whittaker, 1994: 3-4). In a prehistoric context, the list of potential injuries that accompany this activity could have proven fatal. On the basis of these considerations, individual (trial-and-error) learning would seem an inherently risky and costly undertaking in the case of knapping. Of course, the production of handaxes requires the successful execution of extended and strategically organized knapping sequences, and learning to effectively reproduce items that resemble prehistoric handaxes takes months, if not years of practice, even for modern humans (Edwards 2001; Stout 2005). Studies of learning strategies across a diverse range of animals show that greater 
emphasis will be placed on social learning mechanisms whenever individual learning is more costly (e.g., Mineka and Cook, 1988: Chivers and Smith, 1995; Kelley et al., 2003) and this pattern has also been supported experimentally in the case of humans (Mesoudi and O’Brien, 2008).

Given these combined factors, Lycett et al. (2015) recently argued that the costs associated with the knapping of handaxes would encourage the adoption of imitative learning mechanisms, specifically because these would facilitate learning so as to countermand the inherent effects of copying error, but at reduced risk to novice knappers compared with total reliance on individual (trial-and-error) learning and emulation. Lycett et al. (2015) noted that further research was necessary to support this proposition. One pertinent factor here, is that despite the widespread assumption that imitation is a relatively "high fidelity" means of social learning compared even to emulation (e.g., Byrne and Russon, 1998; Tomasello, 1999; Heyes, 2009; Shea, 2009; Whiten et al., 2009) the outcomes of various means of social learning on patterns of artifactual variation are not well studied empirically. If imitation as a mechanism of social learning is no more effective at lowering mutation rates (i.e., copying errors) compared to emulation, then its relevance to the Acheulean is questionable.

As noted earlier, emulation is a mode of social learning that involves copying only the behavioral outcomes or "result(s)" of an another individual's behavior and, therefore, does not involve direct copying of the behavioral actions or "techniques" employed by that individual to bring about that result (Tomasello, 1987; Nagell et al., 1993; Whiten et al., 2004). Emulation has, therefore, also been referred to as "end-state" copying (e.g., Whiten et al., 2009: 2419; Caldwell et al., 2012), which in the case of an artifact would obviously be its overall form and intrinsic properties. Conversely, imitation (Thorndike, 1898) is classed as a distinctive mode of social learning because it also involves the learner directly copying the detailed techniques and behavioral actions of another individual in order to bring about the same "result" (see e.g., Whiten et al., 2004). Given these contrasts, there have been suggestions that imitation will lead to greater fidelity in behavioral outcomes because of its greater capacity for the more "complete" and "accurate" learning of both the manufacturing actions and the actual physical properties of the artifact itself (e.g., Byrne and Russon, 1998; Tomasello, 1999; Heyes, 2009; Shea, 2009; Whiten et al., 2009). Accordingly, emulation has been considered to be relatively poor at maintaining copying fidelity in traditions, and as such, would not (theoretically) have the same capacity to sustain behavioral patterns over the course of time as imitation (Galef, 1992; Tomasello et al., 1993; Tomasello 1999). However, as we have noted, such 
assumptions have not been well tested empirically, especially in terms of how these contrasting learning mechanisms mediate copying error during the manufacture of material artifacts, which is obviously the issue of most relevance when it comes to understanding the factors most likely to have been at work in forming the Acheulean.

Given this state of affairs, we (Schillinger et al., 2015) recently undertook an experiment aimed directly at testing the outcomes of "imitative" versus "emulative" social learning mechanisms on artifactual shape copying error. To implement this experiment, we used the method of copying the form an Acheulean handaxe (Figure 5) from the standardized foam blocks that we described earlier. This method effectively circumvents the health and safety difficulties of asking experimental participants to engage in stone knapping, while also making none of the inherent demands on "skill" or expertise that is required in the production of real handaxes, so enabling the recruitment of a sufficient number $(n=60)$ of participants necessary for robust statistical analysis. Elsewhere, we have noted that this experimental protocol for studying artifact "mutation" (i.e., copy error) is similar in motivation, practical utility, and analytical efficacy to the use of relatively simple "model organisms" (e.g., fruit flies) by experimental biologists to study fundamental factors of organic evolution such as genetic mutation (Schillinger et al., 2014a, 2014b).

We divided the participants into two equal groups of 30, with an even number of males and females in each group (see Schillinger et al., 2015 for full details). The basic task was a simple one: the participants were asked to copy the shape of a foam "target" handaxe (Figure 5) from the standardized block of foam $(22.3 \times 11 \times 7.8 \mathrm{~cm})$ using a plastic table knife to cut and shape the block. The participants in each group were exposed to different learning conditions, consistent with the overall aims of the experiment. Participants in an "emulation" condition were shown only the target "handaxe" prior to beginning the copying task. Conversely, those in the "imitation" condition were additionally shown a short video (4 minutes and 50 seconds) which enabled them to observe the major techniques and sequence of procedural steps that had been used in the manufacture of the original target model. This video was deliberately produced and edited so that prolonged exposure to the final artifact was avoided, thus ensuring that participants in the imitation condition did not observe the target for any longer than participants in the emulation condition prior to beginning the task. Participants in both experimental conditions were provided with one full minute to handle and inspect the model prior to making their copy. During this phase, they were advised to pay attention to 
the overall form, dimensions, and shape properties of the model "handaxe", but were instructed to prioritize copying the shape of the model. In both conditions, the target model remained with the participants at all times, thus controlling for potential memory effects (e.g., Eerkens, 2000). The participants were also permitted to compare their replica with the model at any point they desired while undertaking the task, although use of measuring instruments (e.g. rulers) was prohibited. In both conditions, 20 minutes were allotted in order to complete the task, a timeframe which prior experiments had indicated was ample to conduct the task effectively (see Schillinger et al., 2014b).

Following the experiment, all of the replicas were subjected to morphometric shape analysis (sizeadjusted data), so that copying error in each condition could be compared statistically. A dataset of 42 morphometric variables was obtained for each handaxe (including the target model) using a standardized orientation protocol (Schillinger et al., 2014b, 2015). The measurements obtained via this method take account of artifactual variation in both plan and profile views (Figure 5). Because the primary objective of the experiment was to investigate the effects of contrasting social learning mechanisms on shape properties, all raw data were size-adjusted via the geometric mean method (Jungers et al. 1995; Lycett et al. 2006). Following size-adjustment, shape error was computed for each specimen by simply subtracting the value of its 42 shape variables from the equivalent values obtained for the target model (Schillinger et al., 2015). Finally, mean shape errors were computed for each of the 42 variables for all of the handaxes produced in the two experimental conditions. These 42 mean error rates were then compared for statistically significant differences using a conservative nonparametric Mann-Whitney $U$ test $(\alpha=0.05)$. It was predicted that if the distinct learning conditions in each of the two experimental cohorts were having pertinent effects, then this should result in significantly different rates of copying error in the "handaxes" produced by each group.

Additionally, video data of participants undertaking the task were analyzed, specifically to determine whether participants in the imitation condition employed the behaviors they had seen in the video demonstration to a significantly greater degree than participants in the emulation condition. Thus, the purpose of this analysis was to formally test that any statistical differences in copying error in the two groups could indeed confidently be attributed to differences in the social learning contexts provided to each group. A "copying fidelity" scoring system was developed, which systematically compared the manufacturing behavior exhibited in the demonstration video to that of the participants. The fidelity coding system ranged from $0-7$, with a score of seven indicating an optimal level of 
correspondence with the demonstration video, while zero indicated the lowest level of behavioral correspondence. In essence, this "fidelity" scoring system was based on the combination of three factors: (1) the number of behaviors exhibited by a participant that were also present in the model video, which we termed "matched behaviors"; (2) adherence to sequence; and (3) the number of behaviors exhibited by a participant that did not appear in the video, which we termed "aberrant" behaviors. To test whether there were statistically significant differences in fidelity scores between conditions (i.e., frequencies of the categories), we used a Pearson chi-square test. In addition, we undertook a statistical examination of just the "matched behaviors" (i.e., the first element of the combined scoring system) using a nonparametric Mann-Whitney $U$ test.

Schillinger et al. (2015) found that the extent of shape copying error (Figure 6) was significantly lower in the imitation learning condition compared with the emulation condition $(U=652$; Monte Carlo $\mathrm{p}=0.0383$ ). Hence, consistent with theoretical predictions, the results of this experiment demonstrate that imitative social learning (i.e., copying of behavioral actions used to achieve a goal) can significantly reduce copying errors seen at the artifactual level compared with emulative social learning (i.e., copying just the artifact). Importantly, the results of the video analysis also identified a significant difference in the frequencies of fidelity code scores seen across the two experimental conditions $\left(\chi^{2}=26.065\right.$; Monte Carlo $\left.\mathrm{p}=0.0001\right)$, with $77 \%$ of the emulation cohort achieving scores in the lowest two categories, while only $27 \%$ of the imitation cohort achieved scores in this range. Likewise, the analysis of "matched behaviors" demonstrated that participants in the imitation cohort exhibited manufacturing behaviors corresponding to those seen in the video significantly more so than the participants in the emulation cohort $(U=115$; Monte Carlo $\mathrm{p}=0.0001)$. Hence, the video analysis demonstrated that participants in the imitation condition more closely matched the demonstrated behaviors compared to the emulation cohort.

In sum, Schillinger et al.'s (2015) results demonstrate that imitative social learning conditionswhereby a learner directly utilizes at least some of the techniques and procedures used by another individual - can significantly reduce copying error rates compared to emulative social learning mechanisms. Moreover, the video analysis confirmed that participants in the imitation condition more closely matched the observed behaviors compared to participants in the emulation condition. In other words, this analysis confirmed that differences in social learning mechanism-specifically, copying the behavior of another individual-directly translates into statistically different rates of 
copying error at the artifactual level. It is notable that these differences emerged even despite the simplicity of the task compared with real handaxe manufacture, whereupon the advantages of seeing the behavior of another can reasonably be expected to be less potent. Indeed, it is interesting to note that individuals in the imitative-learning group did not copy the behaviors they had observed in the video perfectly; errors in behavior matching, procedural sequence, and use of aberrant behaviors were all evident (Schillinger et al., 2015). Importantly, however, the results of this experiment demonstrate that even despite imperfect imitation, even flawed copying of the witnessed behaviors still translated into significantly reduced copy-error rates in the artifacts produced. Moreover, these effects were "immediate" in the sense that the participants only had one attempt at the task, which is particularly important in relation to potential effects on copying error in a dangerous learning environment where rapid and effective learning would be most desirable. That is, the experiment shows that even imitating the behavior of another individual with less than $100 \%$ accuracy, has the capacity to more quickly and effectively bring about the appropriate result (i.e., reduced copying error), which would be important in any context where less effective results would have direct, and potentially dangerous, costs.

\section{Discussion}

The foregoing experimental studies illustrate that multiple, opposing factors are likely to have both generated and constrained variability within the Acheulean in terms patterns of handaxe variation. To understand this phenomenon at a broad scale and move forward with further comparative work, what is needed is a framework that can accommodate these multiple, different (microevolutionary) forces, yet also reconcile the multiple facets of the empirical (macroevolutionary) Acheulean record.

Recently, Lycett and von Cramon-Taubadel (2015) have argued that "quantitative genetics" principles, drawn from biology, may be extremely useful in the case of lithic artifacts, directly because of a need to reconcile multiple, and sometimes seemingly contradictory, forces that may act in causing and guiding patterns of variation. The foundation of quantitative genetics stems from a small number of fundamental principles, the primary of which is that the variation exhibited in the physical characteristics (or "traits") of a population of organisms can be modeled as deriving from both genetic (i.e., heritable) and environmental (i.e., nonheritable) components (Falconer and Mackay, 1996; Lynch and Walsh 1998). "Environment" in this instance refers to any factor (e.g., diet, activity patterns, exposure to pathogens) that has an effect on the physical (i.e., phenotypic) differences between individuals other than genetic factors. For instance, two individuals with the 
same genetic disposition for a characteristic such as "height" will obviously not grow to be equally tall if one experiences lengthy bouts of malnutrition during development while the other does not. Moreover, life history or "senescence" effects must also be considered; the length of a bird's beak, for instance, may grow atypically long due to lack of wear in captive situations (Fox, 1952), indicating that extent and pattern of usage is an "environmental" factor that will affect variation within such features. Under a quantitative genetic framework, the total variation $\left(\mathrm{V}_{\mathrm{P}}\right)$ for a measurable, physical trait for any given population would thus be modeled as:

$\mathrm{V}_{\text {Phenotype }}=\mathrm{V}_{\text {Genetic }}+\mathrm{V}_{\text {Environment }}$

where $V_{G}$ is the proportion of the total variation in a given trait controlled by genetic factors, and $V_{E}$ is the proportion of the overall variation caused by environmental factors (see e.g., Falconer, 1960). All that is required for the trait to be amenable to evolutionary forces (e.g., drift or selection) in this model is that some portion of the total variation within the population is at least partly controlled by a heritable (i.e., genetic) component. One factor to note here is that genetic and environmental components of variation are not set up as unconditional alternatives: it is $\mathrm{V}_{\mathrm{G}}$ plus $\mathrm{V}_{\mathrm{E}}$, not $\mathrm{V}_{\mathrm{G}}$ or $\mathrm{V}_{\mathrm{E}}$. In essence, the quantitative genetics framework is simply a way of expressing how both heritable and nonheritable sources of variation can be taken into account as separate subcomponents of the total amount of variation that is physically measurable in a given, empirically observable, trait.

Similarly, in the case of handaxes, multiple specific factors might also cause variation at the assemblage level, and not all of this variation is necessarily heritable in cultural evolutionary systems. Raw material factors, of the type discussed earlier (i.e., internal and external properties of raw materials) would constitute one potential source of "environmental" variation, even within a cultural system where social learning is providing a source of heritable continuity. A further potential source of variability affecting stone tools such as handaxes is resharpening (Frison, 1968; Dibble, 1984; McPherron, 1999). That is, as a stone tool is blunted or damaged through use, subsequent resharpening or retouching might affect the form of lithic artifacts that eventually enter the archaeological record.

Thinking about an entity such as the Acheulean clearly requires a framework that can incorporate and reconcile multiple heritable and nonheritable sources of variation within a single scheme. As Lycett and von Cramon-Taubadel (2015) have noted, however, in the light of a quantitative genetic framework, we can model variation in a set of lithic artifacts as: 
$\mathrm{V}_{\text {ArtifactSet }}=\mathrm{V}_{\text {Culture }}+\mathrm{V}_{\text {RawMaterial }}+\mathrm{V}_{\text {Reduction, }}$

where $\mathrm{V}_{\text {Culture }}$ is the proportion of overall variation within a set, i.e. "population," of artifacts controlled by cultural factors, $\mathrm{V}_{\text {RawMaterial }}$ is the proportion of the variance caused by raw material factors, and $\mathrm{V}_{\text {Reduction }}$ is the proportion of total variation attributable to reduction factors. As in the biological case, all that is required for a trait to be amenable to evolutionary forces (e.g., drift or selection) in this model is that the total variation of any given trait is at least partly controlled by heritable (i.e., socially learned) factors (Figure 7).

It should be noted that $\mathrm{V}_{\text {Culture }}$ can include anything that is inherited by individuals (or is at least potentially heritable) via any mechanism of social learning, consciously or otherwise, such that it comes to propagate and influence the manufacture and final form of artifactual traits. In the case of lithic artifacts, these could include various "process controls" (sensu Patten, 2005: 55; 2012), such as methods of platform preparation, faceting, angle choice, use of soft hammers, etc. They might also be comprised of social conventions that influence the mean size and/or shape of artifacts, either for functional or aesthetic reasons, or simply unconscious convention. Indeed, it is important to stress that while some of these socially learned influences on stone artifact form may be consciously imposed by knappers (as in the use of "process controls"), other socially learned factors that come to influence statistical patterns of variation in different populations of manufacturers may be entirely unconscious in nature. In this sense, statistically identifiable patterns in artifacts may reflect either functional parameters or nonfunctional parameters, or may be the result of conscious or unconscious actions on the part of individuals and communities. There is no stipulation on these matters for a particular statistical pattern to qualify as "cultural" under this framework.

In biology, one of the important implications of a quantitative genetics framework is that it can be used to establish a quantitative concept of "heritability" for empirically measurable traits even though not all of the variability manifest in those traits is actually heritable (Falconer and Mackay, 1996; Roff, 1997; Lynch and Walsh, 1998; Conner and Hartl, 2004). Indeed, the quantitative parameter of "heritability" is the key to understanding, and explaining, evolution given the complexities of continuous data under these conditions (reviewed in Lycett, in press). This is because this parameter relates directly to the role that factors such as selection (of any form) and/or drift might have on the variance and mean value of a trait measured across time or space. In terms of a metric biological 
phenotypic trait (e.g., "length" of a skull, or beak "shape"), the heritability of the trait (notated as $H^{2}$ ) is the proportion of the total phenotypic variance across the population (i.e., $\mathrm{V}_{\mathrm{P}}$ ), expressed as a ratio of the variance attributable to genetic factors (i.e., $\mathrm{V}_{\mathrm{G}}$ ). Hence, simply:

$$
H^{2}=\frac{V_{G}}{V_{P}}
$$

The use of the squared symbol here is a reminder that the parameter of heritability is based on the descriptive statistic of variance (i.e., the standard deviation squared) in terms of the two variables used to compute it. Because the denominator $\left(\mathrm{V}_{\mathrm{P}}\right)$ is inevitably larger than the numerator $\left(\mathrm{V}_{\mathrm{G}}\right)$, heritability values always range between 0 and 1 . (Note, that due to complications that do not concern us here, the parameter described above is strictly referred to as "broad sense" heritability, which is often contrasted with "narrow sense" heritability, or $h^{2}$ in biological usage.)

In quantitative genetic terms, therefore, the "heritability" of a biological quantitative trait is the degree to which variation in that trait (measured across a group of individuals) is determined by genetic factors, expressed as a ratio of the degree to which variation in that trait is also determined by additional (i.e. nonheritable) factors (Lycett, in press). Because total phenotypic variance $\left(\mathrm{V}_{\mathrm{P}}\right)$ is the sum of both genetic and environmental components (i.e., $V_{G}+V_{E}$ ), one of the main factors affecting the value of $H^{2}$ is the extent to which variation in the trait is determined by nonheritable (i.e., “environmental") factors. In cases where environment is having a relatively large effect, the computed value of $H^{2}$ will decrease. If, hypothetically, $H^{2}=0$, then offspring have no heritable means of resembling their parents in that trait and so evolutionary forces (e.g., selection or drift) cannot influence changes in the mean value and/or variance of that trait. In cases where $H^{2}$ has a value approaching 1, selection or drift has the greatest potential to produce change in the mean and/or variance values of that trait in subsequent generations. Understanding this ratio is, therefore, fundamental to understanding the relationship between how things look in one generation, compared to how they might look in the next generation once drift and/or selection has done its work.

Under this framework, the potential capacity for evolutionary change in a given trait can be expressed mathematically by what is referred to as "breeder's equation" (see e.g., Conner and Hartl, 2004). The breeder's equation calculates what is termed the "response to selection" $(R)$, using the "selection differential" $(S)$, which is simply the difference between the mean value of the entire population and the mean of those individuals who are not subject to negative selection. It is, then, computed as: 
$R=H^{2} S$.

The "response to selection" $(R)$ is, therefore, a value that describes the magnitude of evolutionary change in the mean value of a trait, given the heritability of the trait, and the potency of selection (or random sorting in the case of drift) on the parent generation. The computed value obviously increases when either the strength of selection $(S)$ increases, and/or the heritability $\left(H^{2}\right)$ increases. As long as both values are not zero, changes in the mean value of the trait can occur.

The importance of these principles for understanding Acheulean handaxe variation may be illustrated via a simple hypothetical example. Imagine a hypothetical set of 10 Acheulean handaxes that vary in a given quantitative trait, such as "length." Although for the sake of illustration, the size variable of "length" is used in the following computations, the same steps could be undertaken for any quantitative (morphometric) character that varies continuously, including size-adjusted shape variables. Imagine that the mean length of our 10 hypothetical handaxes is $13.35 \mathrm{~cm}$ and the variance $\left(\sigma^{2}\right)$ is 6 . Given the framework outlined earlier, we can therefore compute heritability $\left(H^{2}\right)$ for this set of handaxes as:

$$
H^{2}=\frac{V_{C}}{V_{A S}},
$$

where, as before, $\mathrm{V}_{\text {ArtifactSet }}$ is the total variance of the trait across the artifact set, and $\mathrm{V}_{\text {Culture }}$ is the proportion of that variation that can be attributed to cultural factors. Let us assume that raw material $\left(\mathrm{V}_{\text {RawMaterial }}\right)$ accounts for $30 \%$ of the total variation; it should be noted that this percentage is not entirely inconsistent with real archaeological data given the accuracy with which handaxes can be correctly assigned to their correct localities in multivariate analyses (e.g., Lycett and Gowlett, 2008; Lycett and von Cramon-Taubadel, 2015). Given therefore that $\mathrm{V}_{\text {ArtifactSet }}=6$, we can determine that $\mathrm{V}_{\text {Culture }}=4.2$ (i.e., $70 \%$ of total variation). Given these values, we can compute heritability $\left(H^{2}\right)$ for the trait of "length" in this set of handaxes as:

$$
H^{2}=\frac{V_{C}}{V_{A S}}=\frac{4.2}{6.0}=0.7
$$

Given this heritability value, let us assume, hypothetically, that either selection or random (stochastic) factors cause the largest five handaxes to be preferentially copied. We can thus compute the "selection differential" $(S)$, as the difference between the mean value of the entire population 
$(13.35 \mathrm{~cm})$ and the mean of the five individuals who are not subject to negative selection (say $15.5 \mathrm{~cm})$.

Given these variables, we can compute the response to selection $(R)$ as:

$R=H^{2} S$

$=0.7 \times(15.5-13.35)$

$=0.7 \times 2.15$

$=1.505$

Put another way, we can predict that the mean length in handaxes would increase in the next "generation" by $\sim 1.5 \mathrm{~cm}$ over that exhibited in the previous "generation." If this were repeated over just five generations, the mean length of handaxes would have increased by $7.5 \mathrm{~cm}$; that is from 13.35 to $20.85 \mathrm{~cm}$. This is despite the fact that not all of the variation is controlled by behavioral factors, and that $30 \%$ of the total variance is determined by raw material factors. It is important to note that the change in mean values will not, however, necessarily lead to a change in overall variance levels on the population level. This is because "environmental" factors (in this case, raw material) will continue to exert an effect on variation at the assemblage level, as will copying error, individual skill differences, etc.

We have discussed these issues at some length because the experimental results described in earlier parts of this paper have direct implications for this framework, particularly in terms of thinking about its consequences for understanding variation within the Acheulean. For instance, we have noted that imitation as a mechanism of social learning within handaxe producing populations would reduce the effects of inevitable copying errors that arise in reductive knapping processes, especially traditions involving a specific shape component (Schillinger et al., 2014a, 2015). Moreover, we have noted that in addition to this, the costs (i.e., dangers) of knapping handaxes would have encouraged imitation as a means of increasing the effectiveness of social learning, in order to actively reduce these costs (Lycett et al., 2015). As we have discussed, comparative studies from a diverse range of animal taxa indicate that social learning will increase whenever the costs of asocial learning are higher (e.g., Mineka and Cook, 1988: Chivers and Smith, 1995; Kelley et al., 2003). Under these circumstances, therefore, monitoring the visible payoffs of knapping events by others, and copying successful outcomes would have been an optimal strategy. Indeed, the copying of what Patten (2005: 55; 2012) refers to as "process controls," i.e., behaviors that lead to an improved consistency of knapping outcomes, would have made particularly effective targets for imitation under such conditions. In 
quantitative genetic terms, what this would actually have done, is that relative to the effects of raw material, the overall proportion of variation within the assemblage controlled by behavioral factors would be increased; in other words, heritability $\left(H^{2}\right)$ increases overall via imitation. This is also consistent with the results of our experimental study examining the effects of raw material on lithic artifact variation (Eren et al., 2014). As we have stressed, it is not that raw material should be considered to exert no potential effect, simply that the capacity for behavior to overcome raw material "constraints" is potentially strong. One implication here is that rather than merely discussing raw material as an absolute determining factor, it is that the relative proportion of variability due to socially learned factors as opposed to raw material factors becomes the more important issue. This could facilitate a new look at spatial and temporal patterns in Acheulean handaxe variation; for instance, whether such proportional relationships change over the timespan of the Acheulean, as the imitative learning of new practices (e.g., new platform preparation techniques and/or the use of soft hammers) increases (see e.g., Stout et al., 2014). In other words, a prediction might be made that the overall relative contribution of raw material to total variation in handaxe assemblages might change over the course of the Acheulean, as a direct result of cultural and biological change over the million (plus) years of its timeframe.

Equally, however, the experimental studies we have discussed have indicated something of the inherent instability of handaxe traditions, as a direct result of the ever present factor of copying error (Kempe et al., 2012; Schillinger et al., 2014a, 2014b). Indeed, even in our study of raw material, using just a single expert knapper, copying error was present even though this was not systematically determined by raw material (Eren et al., 2014); in other words, even at the level of an expert individual, no handaxe was an exact copy of another. This does, however, bring the major question regarding the "stability" of the "Acheulean" back full circle. Indeed, as our discussion of the parameter of "heritability" demonstrates, in a situation where imitation is providing a means of social replication, yet sources of variation (i.e., artifactual mutation via copying errors) are ever present, then, profound change via drift and/or or selective "radiation" of new tool forms would be a predicted outcome (see e.g., Kempe et al., 2014), precisely due to the evolutionary mechanisms that we have outlined. Indeed, the combination of high mutation and high potential for variation to be heritable would lead to what biologists call a high capacity for "evolvability" (e.g., Ridley 2004: 587). 
The paradoxical character of the Acheulean as a feature of the Pleistocene that exhibits both stability and variability has been discussed on many occasions. Glynn Isaac's phrase of "variable sameness" (see e.g., Gowlett et al., 2001: 612) captures the fact that examination of the "Acheulean" requires discussion of both continuity within a framework that also adequately account for capriciousness. "Variability" at the level of the Pleistocene, however, extends beyond merely variation within and between assemblages of handaxes to a phenomenon that even demands the explanation of the "absence" of handaxes, as is the case with the Clactonian of Britain or the "patchiness" of handaxe production east of the so-called "Movius Line." Indeed, concepts of "variability" over the course of the Pleistocene might also need to take into consideration long-term trends that take place, such as changes in the form and symmetry that some argue to occur during the "Late Acheulean" (e.g., Clark, 1994; Wynn, 2002; Beyenne et al., 2013; Stout et al., 2014). Hence, "the Acheulean" is comprised of an amalgam of long-term, short-term, local, and continental trends, all of which need to be recognized and accounted for. To maintain functional integrity of the handaxe "bauplan" (sensu Lycett and Gowlett, 2008) there are obviously limits to artifactual variability (Figure 8). The experiments described earlier indicate that imitation was a mechanism by which this integrity of form (as attested archaeologically by the "Acheulean") was maintained, yet also highlight the role of copying errors, the presence of which provided necessary fuel for either drift or selection over intermediate spans of space and time (Figure 8). This pattern is what Isaac (1972) referred to as the "random walk" of Acheulean variation, or what more recently Crompton and Gowlett (1993: 177) termed a zone of "free play" within which handaxe traits may vary. It is important to note, however, that it would be possible for some traits within this framework to be under either long-term or short term selective pressures, while still other traits were undergoing drift (Lycett, 2008). Indeed, this pattern may actually be apparent in a range of functional lithic technologies, and resembles the "wiggle room" that has recently been discussed in the case of Clovis projectile technologies in North America (Eren et al., 2014: 168).

While many aspects of this explanatory framework (Figure 8) for Acheulean variation are not therefore new, experiments of the type we have outlined, and the microevolutionary perspective they provide, give new and more precise insights into the mechanisms that underlie this pattern. Indeed, the role that they play in highlighting the "stabilizing" effect of imitative learning mechanisms in the face of a steady mutation rate caused by inevitable copying errors, emphasizes the importance of skilled knappers being present within "Acheulean" hominin communities in terms of continuity of tradition. In a situation where mutation leads to a tendency toward disintegration, expansions into 
new areas would have implications for "cultural bottlenecks" resulting from founder effects, such as may occur at the limits of Acheulean expansion, for instance in Britain and East Asia (Lycett and von Cramon-Taubadel, 2008). Indeed, the role of founder effects resulting from dispersal, topographical barriers, and/or ecological factors (Lycett and von Cramon-Taubadel, 2008; Smith et al., 2009; Lycett and Norton, 2010) is re-emphasized in the light of our experiments on imitation.

\section{Conclusion}

The so-called "Acheulean" represents a pattern of repeated hominin behavior (biface production) over wide spans of space and time. It correctly attracts our attention because, as such, it is a major, macroscale feature that provides both challenge and opportunity in terms of a means of comparative behavioral analysis, and in terms of demanding explanation of all its vagaries, including the "spaces" and vicissitudes of variability that take place within it. Any major archaeological patterns that we see on a temporal and/or geographical level, however, inevitably begin at the level of the individual - the individual artifact and the individual knapper. Understanding microevolutionary factors will, therefore, be essential in understanding the macroscale patterns we observe. Here, we have synthesized a range of experimental results that provide insights into the microevolutionary processes likely to have played a role in constituting the Acheulean record. These experiments provide a new perspective on the role of issues such as raw material, copying errors, and social learning mechanisms and how these will have interacted to constitute the long-term, short-term, geographically widespread, and localized patterns that are conflated under the term "Acheulean." In other words, they give insights into the precise factors that both generate and constrain variation at a multiplicity of scales. In the future, we contend, further experiments of this type will, therefore, form an essential role in adequately analyzing and understanding the phenomenon we have traditionally referred to as "the Acheulean," especially when combined with the flexibility provided by a "quantitative genetic" approach to the cultural evolution of such phenomena. 


\section{References}

Ambrose, S.H., 2001. Paleolithic technology and human evolution. Science 291, 1746-1753.

Andrefsky, W., 1998. Lithics: Macroscopic Approaches to Analysis. Cambridge University Press, Cambridge.

Ashton, N., McNabb, J., 1994. Bifaces in perspective, in: Ashton, N., David, A. (Eds.), Stories in Stone: Lithic Studies Society Occasional Paper No.4. Lithic Studies Society, London, pp. 182-191.

Baumler, M.F., 1995. Principles and properties of lithic core reduction: implications for Levallois technology. In: Dibble, H.L., Bar-Yosef, O. (Eds.), The Definition and Interpretation of Levallois Technology. Prehistory Press, Madison, Wisconsin, pp.11-23.

Bello, S.M., Parfitt, S.A., Stringer, C., 2009. Quantitative micromorphological analyses of cut marks produced by ancient and modern handaxes. Journal of Archaeological Science 36, 1869-1880

Beyene, Y., Katoh, S., WoldeGabrield, G., Hart, W.K., Uto, K., Sudo, M., Kondo, M., Hyodo, M., Renne, P., Suwa, G., Asfaw, B., 2013. The characteristics and chronology of the earliest Acheulean at Konso, Ethiopia. Proceedings of the National Academy of Sciences 110 (5), 1584-1591.

Bookstein, F.L., 1989. 'Size and shape': a comment on semantics. Systematic Zoology 38, 173-180.

Boyd, R., Richerson, P., 1985. Culture and the Evolutionary Process. University of Chicago Press, Chicago.

Buchanan, B., O'Brien, M., Collard, M., 2014. Continent-wide or region-specific? A geometric morphometrics-based assessment of variation in Clovis point shape. Archaeological and Anthropological Sciences 6, 145-162.

Byrne, R.W., Russon, A.E. 1998. Learning by imitation: a hierarchical approach. Behavioral and Brain Sciences, 21, 667-721.

Caldwell, C.A., Schillinger, K., Evans, C.L., Hopper, L.M. 2012. End state copying by humans (Homo sapiens): implications for a comparative perspective on cumulative culture. Journal of Comparative Psychology, 126, 161-169.

Callahan, E., 1979. The basics of biface knapping in the eastern fluted point tradition: a manual for flintknappers. Archaeology of Eastern North America 7, 1-180.

Cavalli-Sforza, L.L., Feldman, M.W., 1981. Cultural Transmission and Evolution: A Quantitative Approach. Princeton University Press, Princeton, NJ.

Chauhan, P.R. 2010. Metrical variability between South Asian handaxe assemblages: preliminary observations. In: Lycett, S.J., Chauhan, P.R. (Eds) New Perspectives on Old Stones (pp. 119-166). Springer New York. 
Chivers, D.P., and R.J.F. Smith 1995 Chemical recognition of risky habitats is culturally transmitted among fathead minnows, Pimephales promelas (Osteichthyes, Cyprinidae). Ethology 99: 286-296.

Clark, J.D., 1994. The Acheulian industrial complex in Africa and elsewhere, in: Corruccini, R.S., Ciochon, R.L. (Eds.), Integrative Paths to the Past. Prentice Hall, Englewood Cliffs, NJ, pp. 451-469.

Clark, J.D., 2001. Variability in primary and secondary technologies of the Later Acheulian in Africa, in: Milliken, S., Cook, J. (Eds.), A Very Remote Period Indeed: Papers on the Palaeolithic Presented to Derek Roe. Oxbow Books, Oxford, pp. 1-18.

Clarkson, C., 2010. Regional diversity within the core technology of the Howiesons Poort technocomplex, in: Lycett, S.J., Chauhan, P.R. (Eds.), New Perspectives on Old Stones: Analytical Approaches to Paleolithic Technologies. Springer, New York, pp. 43-59.

Conner, J.K., Hartl, D.L., 2004. A Primer of Ecological Genetics. Sinauer, Sunderland, MA.

Costa, A.G., 2010. A geometric morphometric assessment of plan shape in bone and stone Acheulean bifaces from the Middle Pleistocene site of Castel di Guido, Latium, Italy, in: Lycett, S.J., Chauhan, P.R. (Eds.), New Perspectives on Old Stones: Analytical Approaches to Paleolithic Technologies. Springer, New York, pp. 23-41.

Crompton, R.H., Gowlett, J.A.J., 1993. Allometry and multidimensional form in Acheulean bifaces from Kilombe, Kenya. Journal of Human Evolution 25, 175-199.

Deetz, J., 1967. Invitation to Archaeology. Natural History Press, Garden City, NY.

Dibble, H.L., 1984. Interpreting typological variation of Middle Palaeolithic scrapers: function, style, or sequence of reduction. Journal of Field Archaeology 11: 431-436.

Diez-Martín, F., Eren, M., 2012. The early Acheulean in Africa. In: Domínguez- Rodrigo, M. (Ed.), Stone Tools and Fossil Bones. Debates in the Archaeology of Human Origins. Cambridge University Press, New York, pp. 310-358.

Domínguez-Rodrigo, M., Serralllonga, J., Juan-Tresserras, J., Alcala, L., Luque, L., 2001. Woodworking activities by early humans: a plant residue analysis on Acheulian stone tools from Peninj (Tanzania). Journal of Human Evolution 40, 289-299.

Domínguez-Rodrigo, M., Diez-Martín, F., Mabulla, A., Baquedano, E., Bunn, H. T., Musiba, C. 2014. The evolution of hominin behavior during the Oldowan-Acheulean transition: Recent evidence from Olduvai Gorge and Peninj (Tanzania). Quaternary International, 322, 1-6.

Edwards, S.W., 2001. A modern knapper's assessment of the technical skills of the Late Acheulean biface workers at Kalambo Falls, in: Clark, J.D. (Ed.), Kalambo Falls Prehistoric Site: Volume III. Cambridge University Press, Cambridge, pp. 605-611.

Eerkens, J.W., 2000. Practice makes within 5\% of perfect: visual perception, motor skills, and memory in artifact variation. Current Anthropology 41, 663-668. 
Eerkens, J.W., Lipo, C.P., 2005. Cultural transmission, copying errors, and the generation of variation in material culture and the archaeological record. Journal of Anthropological Archaeology 24, 316334.

Eren, M.I., Lycett, S.J., Roos, C., Sampson, C.G., 2011. Toolstone constraints on knapping skill: Levallois reduction with two different raw materials. Journal of Archaeological Science 38, 27312739.

Eren, M.I., Roos, C.I., Story, B., von Cramon-Taubadel., N., Lycett, S.J. 2014. The role of raw material differences in stone tool shape variation: an experimental assessment. Journal of Archaeological Science 49: 472-487.

Eren, M. I., Buchanan, B., O'Brien, M. J. 2015. Social learning and technological evolution during the Clovis colonization of the New World. Journal of Human Evolution, 80, 159-170.

Falconer, D. S. 1960. Introduction to Quantitative Genetics. New York: Ronald Press Co.

Falconer, D. S., Mackay T. F. C. 1996. Introduction to Quantitative Genetics (Fourth edition). Harlow: Pearson.

Falsetti, A.B., Jungers, W.L., Cole III, T.M., 1993. Morphometrics of the Callitrichid forelimb: a case study in size and shape. International Journal of Primatology 14, 551-572.

Fox, W. 1952. Behavioral and evolutionary significance of the abnormal growth of beaks of birds. Condor 54 (3), 160-162.

Frison, G. C. 1968. A functional analysis of certain chipped stone tools. American Antiquity 33, 149155.

Galef, B.G. 1992. The question of animal culture. Human Nature, 3, 157-178.

Goodman, M.E., 1944. The physical properties of stone tool materials. American Antiquity 9, 415433.

Gowlett, J.A.J., 2006. The elements of design form in Acheulian bifaces: modes, modalities, rules and language. In: Goren-Inbar, N., Sharon, G. (Eds.), Axe Age: Acheulian Tool-making from Quarry to Discard. Equinox, London, pp. 203-221.

Gowlett, J.A.J., 2011. The Empire of the Acheulean strikes back. In: Sept, J., Pilbeam, D. (Eds.), Casting the Net Wide: Papers in Honor of Glynn Isaac and His Approach to Human Origins Research. American School of Prehistoric Research, Cambridge, MA, pp. 93-114.

Hamilton, M.J., Buchanan, B., 2009. The accumulation of stochastic copying errors causes drift in culturally transmitted technologies: quantifying Clovis evolutionary dynamics. Journal of Anthropological Archaeology 28, 55-69. 
Hampton, O.W., 1999. Culture of Stone: Sacred and Profane Uses of Stone among the Dani. Texas A\&M, College Station.

Haslam, M., Roberts, R. G., Shipton, C., Pal, J. N., Fenwick, J. L., Ditchfield, P., Boivin, N., Dubey, A.K., Gupta, M.C., Petraglia, M. 2011. Late Acheulean hominins at the Marine Isotope Stage 6/5e transition in north-central India. Quaternary Research, 75 (3), 670-682.

Heyes, C. 2009. Evolution, development and intentional control of imitation. Philosophical Transactions of the Royal Society B: Biological Sciences, 364, 2293-2298.

Hou, Y., Potts, R., Baoyin, Y., Zhengtang, G., Deino, A., Wei, W., Clark, J., Guangmao, X., Weiwen, H., 2000. Mid-Pleistocene Acheulean-like stone technology of the Bose Basin, South China. Science 287, 1622-1626.

Isaac, G.L., 1972. Early phases of human behaviour: models in Lower Palaeolithic archaeology, in: Clarke, D.L. (Ed.), Models in Archaeology. Methuen, London, pp. 167-199.

Isaac, G.L., 1977. Olorgesailie: Archaeological Studies of a Middle Pleistocene Lake Basin in Kenya. University of Chicago Press, Chicago.

Isaac, G.L. 1983. Aspects of human evolution. In: Bendall, D.S. (Ed.) Evolution from Molecules to Men. Cambridge University Press, Cambridge, pp.509-543.

Jennings, T.A., Pevny, C.D., Dickens, W.A., 2010. A biface and blade core efficiency experiment: implications for Early Paleoindian technological organization. . Journal of Archaeological Science $37,2155-2164$.

Jones, P.R., 1979. Effects of raw material on biface manufacture. Science 204, 835-836.

Jones, P.R., 1980. Experimental butchery with modern stone tools and its relevance for Palaeolithic archaeology. World Archaeology 12, 153-165.

Jordan, P., 2015. Technology as Human Social Tradition: Cultural Transmission among HunterGatherers. University of California Press, Berkeley.

Jungers, W.L., Falsetti, A.B., Wall, C.E., 1995. Shape, relative size, and size adjustments in morphometrics. Yearbook of Physical Anthropology 38, 137-161.

Kelley, J.L., J.P. Evans, I.W. Ramnarine, and A.E. Magurran 2003 Back to school: can antipredator behaviour in guppies be enhanced through social learning? Animal Behaviour 65:655-662.

Kempe, M., Lycett, S.J., Mesoudi, A. 2012. An experimental test of the accumulated copying error model of cultural mutation for Acheulean handaxe size. PLoS ONE, 7, e48333.

Kempe, M., Lycett, S.J., Mesoudi, A. 2014. Cultural differences and cumulative culture: parameterizing the differences between human and nonhuman culture. Journal of Theoretical Biology $359,29-36$. 
Kroeber, T. 1961 Ishi in Two Worlds. University of California Press, Berkeley.

Lepre, C.J., Roche, H., Kent, D.V., Harmand, S., Quinn, R.L., Brugal, J.-P., Texier, P.-J., Lenoble, A., Feibel, C.S., 2011. An earlier origin for the Acheulian. Nature 477, 82-85.

Li, H., Li, C. R., Kuman, K., Cheng, J., Yao, H. T., Li, Z. 2014. The Middle Pleistocene handaxe site of Shuangshu in the Danjiangkou Reservoir Region, central China. Journal of Archaeological Science, 52, 391-409.

Lynch, M., Walsh, B. 1998. Genetics and Analysis of Quantitative Traits. Sunderland: Sinauer.

Lycett, S.J., 2008. Acheulean variation and selection: does handaxe symmetry fit neutral expectations? Journal of Archaeological Science 35, 2640-2648.

Lycett, S.J. 2015. Cultural evolutionary approaches to artifact variation over time and space: basis, progress, and prospects. Journal of Archaeological Science, 56, 21-31.

Lycett, S.J., Bae, C.J., 2010. The Movius Line controversy: The state of the debate. World Archaeology 42, 521-544.

Lycett, S.J., Norton, C.J., 2010. A demographic model for Palaeolithic technological evolution: the case of East Asia and the Movius Line. Quaternary International 211, 55-65.

Lycett, S.J., Gowlett, J.A.J., 2008. On questions surrounding the Acheulean tradition. World Archaeology 40, 295-315.

Lycett, S.J., von Cramon-Taubadel, N., 2008. Acheulean variability and hominin dispersals: a modelbound approach. Journal of Archaeological Science 35, 553-562.

Lycett, S.J., von Cramon-Taubadel, N., Foley, R.A. 2006. A crossbeam co-ordinate caliper for the morphometric analysis of lithic nuclei: a description, test and empirical examples of application. Journal of Archaeological Science 33, 847-861.

Lycett, S.J., von Cramon-Taubadel, N. 2015. Toward a quantitative "genetic approach" to lithic variation. Journal of Archaeological Method and Theory. DOI: 10.1007/s10816-013-9200-9

Lycett, S.J., Schillinger, K., Kempe, M., Mesoudi, A. 2015. Approaching learning in the Acheulean: insights from experiments using handaxe form as a "model organism." In: A. Mesoudi \& K. Aoki (Eds.), Learning strategies and cultural evolution during the Paleolithic (pp. 155-166). New York: Springer.

Mace, R., Pagel, M., 1997. Phylogenies and cultural evolution. Evolution and Human Behavior 18, 349-351.

McPherron, S.P., 1999. Ovate and pointed handaxe assemblages: two points make a line. Préhistoire Européenne 14: 9-32. 
Mesoudi, A., 2011. Cultural Evolution: How Darwinian Theory can Explain Human Culture \& Synthesize the Social Sciences. Chicago University Press, Chicago.

Mesoudi, A., O'Brien, M.J., 2008. The cultural transmission of Great Basin projectile point technology I: An experimental simulation. American Antiquity 73, 3-28.

Mesoudi, A., Whiten, A. 2008. The multiple roles of cultural transmission experiments in understanding human cultural evolution. Philosophical Transactions of the Royal Society B, 363, 3489-3501.

Mesoudi, A., Whiten, A., Laland, K.N., 2004. Is human cultural evolution Darwinian? Evidence reviewed from the perspective of The Origin of Species. Evolution 58, 1-11.

Mineka, S., Cook, M., 1988. Social learning and the acquisition of snake fear in monkeys. In: Galef, B.G., Zentall, T.R. (Eds) Social learning: Psychological and Biological Perspectives. Erlbaum, Hillsdale NJ, pp.51-73.

Mithen, S. 1999. Imitation and cultural change: a view from the Stone Age, with specific reference to the manufacture of handaxes. In: H.O. Box \& K.R. Gibson (Eds.), Mammalian social learning: comparative and ecological perspectives (pp. 389-99). Cambridge University Press, Cambridge.

Noll, M.P., Petraglia, M.D., 2003. Acheulean bifaces and early human behavioral patterns in East Africa and South India. In: Soressi, M., Dibble, H.L. (Eds.), Multiple Approaches to the Study of Bifacial Technologies. University of Pennsylvania, Philadelphia, PA, pp. 31-53.

Norton, C. J., Bae, K. 2009. The Movius Line sensu lato further assessed and defined. Journal of Human Evolution, 57(3), 331-334.

Norton, C.J., Bae, K., Harris, J.W.K., Lee, H., 2006. Middle Pleistocene handaxes from the Korean Peninsula. Journal of Human Evolution 51, 527-536.

Nunn, C. L., 2011. The Comparative Approach in Evolutionary Anthropology and Biology. University of Chicago Press, Chicago.

O’Brien, M.J., Lyman, R.L., 2000. Applying Evolutionary Archaeology: A Systematic Approach. Kluwer Academic / Plenum, New York.

Patten, B., 2005. Peoples of the Flute: A Study in Anthropolithic Forensics. Stone Dagger Publications, Denver, CO.

Patten, B., 2012. Explaining temporal change in artifacts by the use of process controls. Lithic Technology 37: 25-34.

Petraglia, M.D., Shipton, C. 2009. Large cutting tool variation west and east of the Movius Line. Journal of Human Evolution, 57: 326-30.

Pope, S.T. 1918. Yahi Archery. University of California Press, Berkeley. 
Ridley, M. 2004. Evolution. 3rd ed. Blackwell, Oxford

Roberts, M.B., Parfitt, S., 1999. Boxgrove: A Middle Pleistocene Hominid Site at Eartham Quarry, Boxgrove, West Sussex. English Heritage, London.

Roche, H., 2005. From simple flaking to shaping: stone-knapping evolution among early hominins. In: Roux, V., Bril, B. (Eds.), Stone Knapping: the Necessary Conditions for a Uniquely Hominin Behaviour. McDonald Institute Monographs, Cambridge, pp. 35-48.

Roe, D.A., 1994. A metrical analysis of selected sets of handaxes and cleavers from Olduvai Gorge, in: Leakey, M.D., Roe, D.A. (Eds.), Olduvai Gorge: Volume 5. Cambridge University Press, Cambridge, pp. 146-234.

Roff, D.A. 1997. Evolutionary Quantitative Genetics. New York: Chapman \& Hall.

Rogers, M. J., Semaw, S. 2009. From nothing to something: the appearance and context of the earliest archaeological record. In Chauhan, P.R. (Ed) Sourcebook of Paleolithic Transitions (pp. 155171). Springer New York.

Roux, V., Brill, B. 2005. Stone Knapping: The Necessary Conditions for a Uniquely Hominin Behaviour. McDonald Institute Monographs, Cambridge.

Schick, K.D., 1994. The Movius line reconsidered, in: Corruccini, R.S., Ciochon, R.L. (Eds.), Integrative Paths to the Past. Prentice Hall, Englewood Cliffs, NJ, pp. 569-596.

Schick, K., 1998. A comparative perspective on Paleolithic cultural patterns. In: Akazawa, T., Aoki, K., Bar-Yosef, O. (Eds.), Neandertals and Modern Humans in Western Asia. Plenum Press, New York, pp. 449-460.

Schick, K., Toth, N., 2006. An overview of the Oldowan industrial complex: the sites and nature of their evidence, in: Toth, N., Schick, K. (Eds.), The Oldowan: Case Studies into the Earliest Stone Age. Stone Age Institute Press, Gosport, IN pp. 3-42.

Schillinger, K., Mesoudi, A., Lycett, S.J. 2014a. Copying error and the cultural evolution of "additive" vs. "reductive" material traditions: an experimental assessment. American Antiquity 79 (1), 128-143.

Schillinger, K., Mesoudi, A., Lycett, S.J. 2014b. Considering the role of time budgets on copy-error rates in material culture traditions: an experimental assessment. PLoS ONE 9 (5) e97157, 1-10.

Schillinger, K., Mesoudi, A., Lycett, S.J. 2015. The impact of imitative versus emulative learning mechanisms on artifactual variation: implications for the evolution of material culture. Evolution and Human Behavior, in press, http://dx.doi.org/10.1016/j.evolhumbehav.2015.04.003

Semaw, S., 2000. The world's oldest stone artefacts from Gona Ethiopia: their implications for understanding stone technology and patterns of human evolution between 2.6-1.5 million years ago. Journal of Archaeological Science 27, 1197-1214. 
Shea, N. (2009). Imitation as an inheritance system. Philosophical Transactions of the Royal Society $B, 364,2429-2443$.

Sharon, G., 2008. The impact of raw material on Acheulian large flake production. Journal of Archaeological Science, 35, 1329-1344.

Shennan, S., 2011. Descent with modification and the archaeological record. Philosophical Transactions of the Royal Society B 366, 1070-1079.

Shipman, P., Walker, A., 1989. The costs of becoming a predator. Journal of Human Evolution 18, 373-392.

Shumaker, R.W., Walkup, K.R., Beck, B.B. 2011. Animal Tool Behavior: The Use and Manufacture of Tools by Animals. John Hopkins University Press.

Simão, J., 2002. Tools evolve: the artificial selection and evolution of Paleolithic stone tools. Behavioral and Brain Sciences 25, 419.

Smallwood, A., 2010. Clovis biface technology at the Topper site, South Carolina: evidence for variation and technological flexibility. Journal of Archaeological Science 37, 2413-2425.

Smallwood, A., 2012. Clovis technology and settlement in the American Southeast: using biface analysis to evaluate dispersal models. American Antiquity 77, 689-713.

Smith, S., Hughes, J., Mithen, S. 2009. Explaining global patterns in Lower Palaeolithic Technology: simulations of hominin dispersal using stepping out. In: Shennan, S. (Ed) Pattern and Process in Cultural Evolution. Berkeley, University of California Press.

Solodenko, N., Zupancich, A., Cesaro, S.N., Marder, O., Lemorini, C., Barkai, R., 2015. Fat residue and use-wear found on Acheulian biface and scraper associated with butchered elephant remains at the site of Revadim, Israel. PLoS ONE 10 (3): e0118572.

Stout, D., 2005. The social and cultural context of stone knapping skill acquisition, in: Roux, V., Bril, B. (Eds.), Stone knapping: the necessary conditions for a uniquely hominin behaviour. McDonald Institute for Archaeological Research, Cambridge, pp. 331-340.

Stout, D., Apel, J., Commander, J., Roberts, M. 2014. Late Acheulean technology and cognition at Boxgrove, UK. Journal of Archaeological Science, 41, 576-590.

Thorndike, E.L. (1898). Animal intelligence: an experimental study of the associative processes in animals. The Psychological Review: Monograph Supplements, 2, 1-109.

Tomasello, M., 1999. The Cultural Origins of Human Cognition. Harvard University Press, Cambridge, MA.

Tomasello, M., Kruger, A.C., Ratner, H.H. 1993. Cultural learning. Behavioral and Brain Sciences, 16, 495-552. 
Vaughan, C.D., 2001. A million years of style and function: regional and temporal variation in Acheulean handaxes, in: Hurt, T.D., Rakita, G.F.M. (Eds.), Style and Function: Conceptual Issues in Evolutionary Archaeology. Bergin \& Garvey, Westport, Connecticut, pp. 141-163.

Wang, W., Lycett, S.J., Cramon-Taubadel, N., Jin, J.J.H., Bae, C.J., 2012. Comparison of handaxes from Bose Basin (China) and the western Acheulean indicates convergence of form, not cognitive differences. PLoS ONE 7 (4), 1-7.

Wang, W., Bae, C.J., Huang, S., Huang, X., Tian, F., Mo, J., Huang, Z., Huang, C., Xie, S., Li, D., 2014. Middle Pleistocene bifaces from Fengshudao (Bose Basin, Guangxi, China). Journal of Human Evolution 69, 110-122.

Whiten, A., Horner, V., Litchfield, C.A., Marshall-Pescini, S., 2004. How do apes ape? Learning \& Behavior 32, 36-52.

Whiten, A., McGuigan, N., Marshall-Pescini, S., Hopper, L.M. 2009. Emulation, imitation, overimitation and the scope of culture for child and chimpanzee. Philosophical Transactions of the Royal Society B, 364, 2417-2428

Whittaker, J.C., 1994. Flintknapping: Making and Understanding Stone Tools. University of Texas Press, Austin.

Wynn, T., 1995. Handaxe enigmas. World Archaeology 27, 10-24.

Wynn, T., 2002. Archaeology and cognitive evolution. Behavioural and Brain Sciences 25, 389-402.

Wynn, T., Tierson, F., 1990. Regional comparison of the shapes of later Acheulean handaxes. American Anthropologist 92, 73-84.

Yravedra, J., Domínguez-Rodrigo, M., Santonja, M., Pérez-González, A., Panera, J., Rubio-Jara, S., Baquedano, E., 2010. Cut marks on the Middle Pleistocene elephant carcass of Áridos 2 (Madrid, Spain). Journal of Archaeological Science 37, 2469-2476. 


\section{Figures}

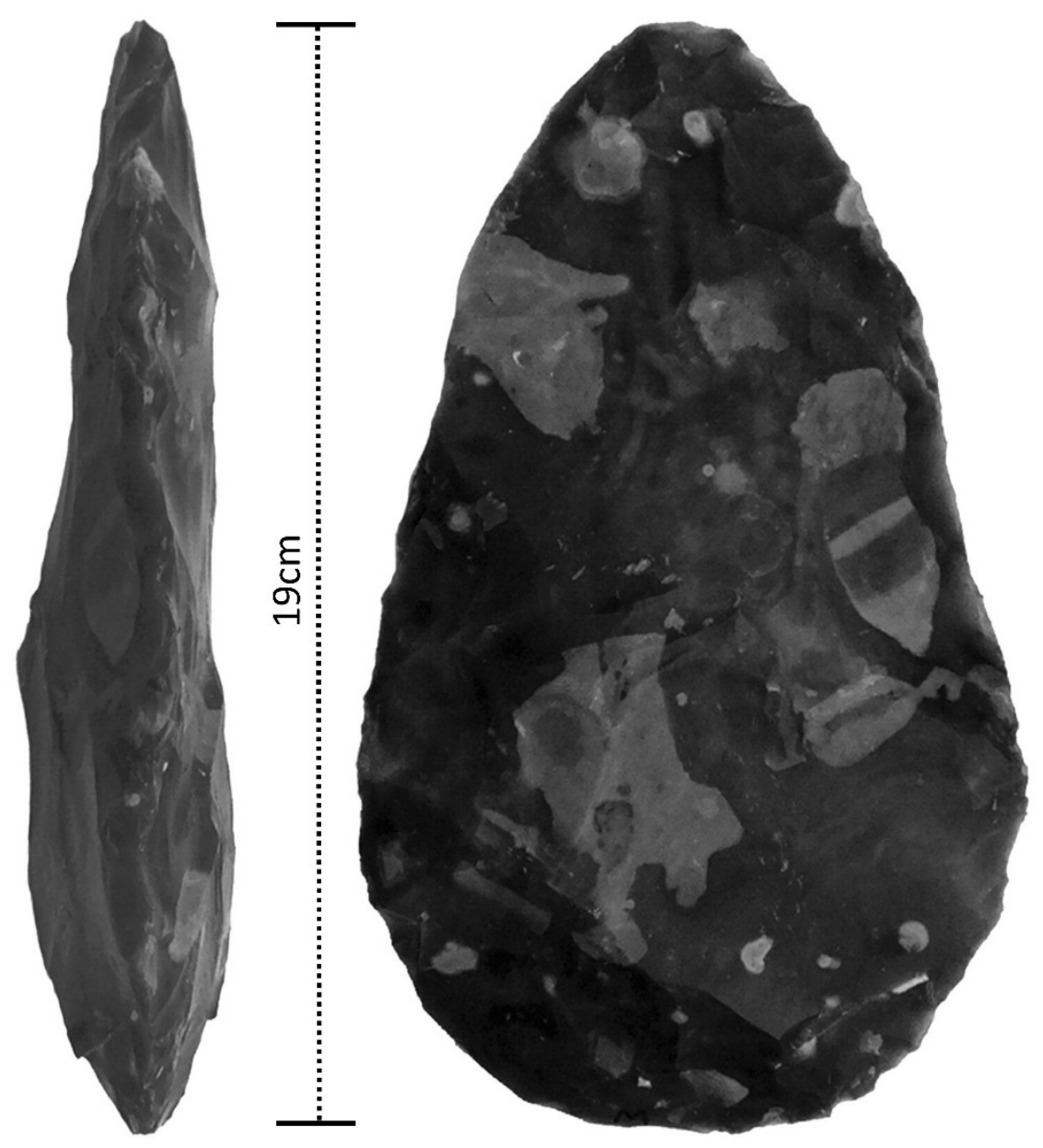

Width/thickness ratio $=3.33: 1$

Figure 1: The "target" model handaxe, which the expert knapper aimed to copy in flint, basalt, and obsidian raw materials (from Eren et al. 2014). 

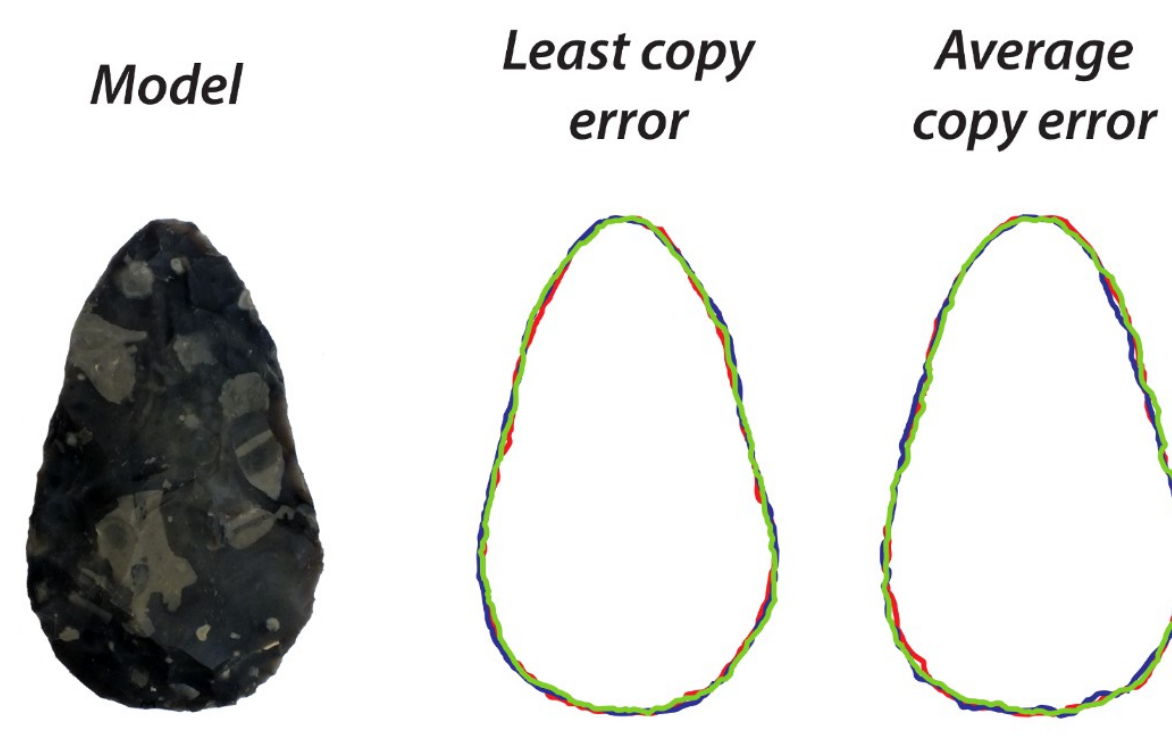

\section{Most copy error}
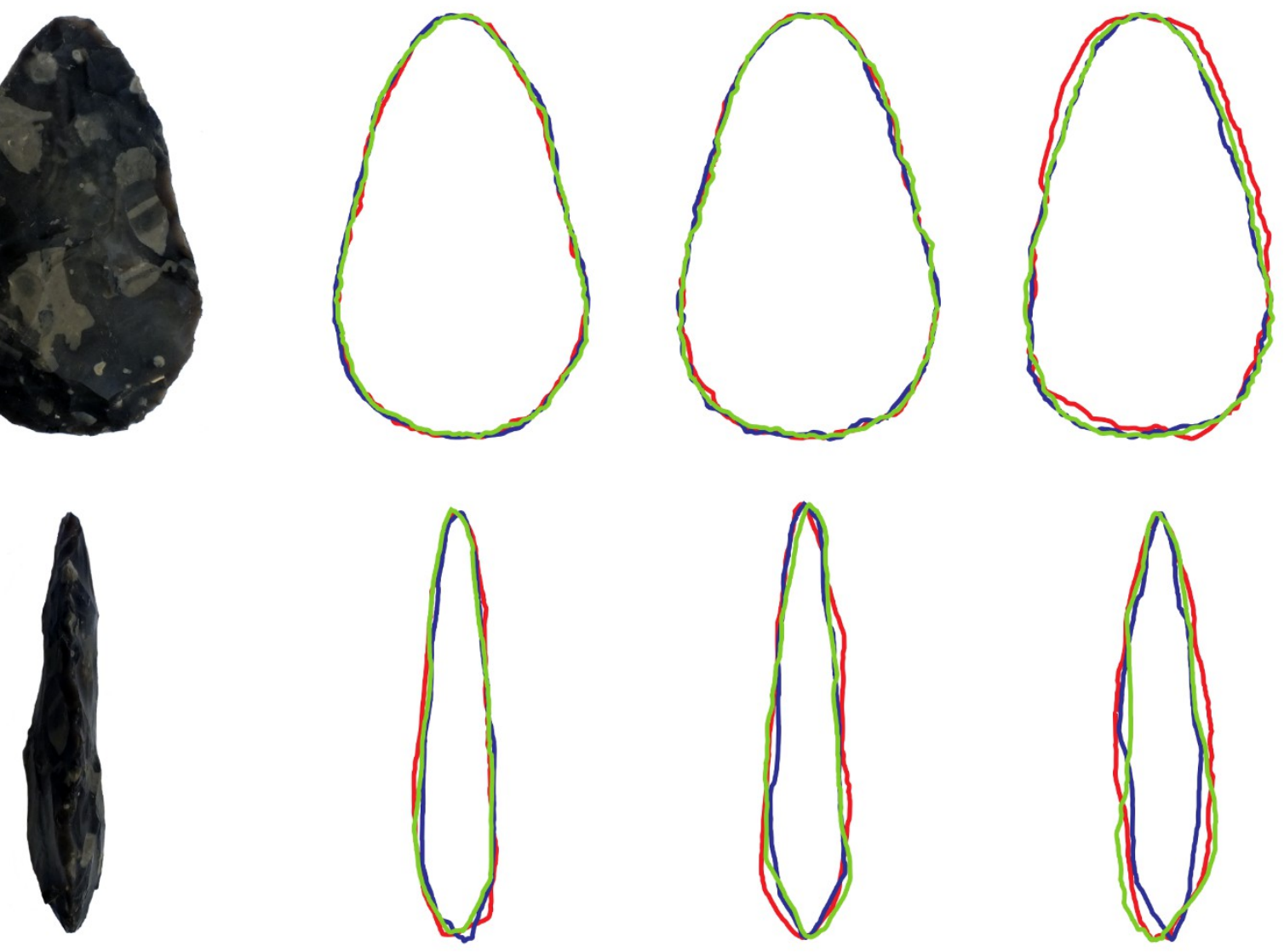

Figure 2: Plan (upper row) and profile (lower row) comparisons of the model "target" handaxe to the least, average, and most copy error handaxes in the flint (red outline), basalt (blue outline), and obsidian (green outline) raw materials (from Eren et al. 2014). 


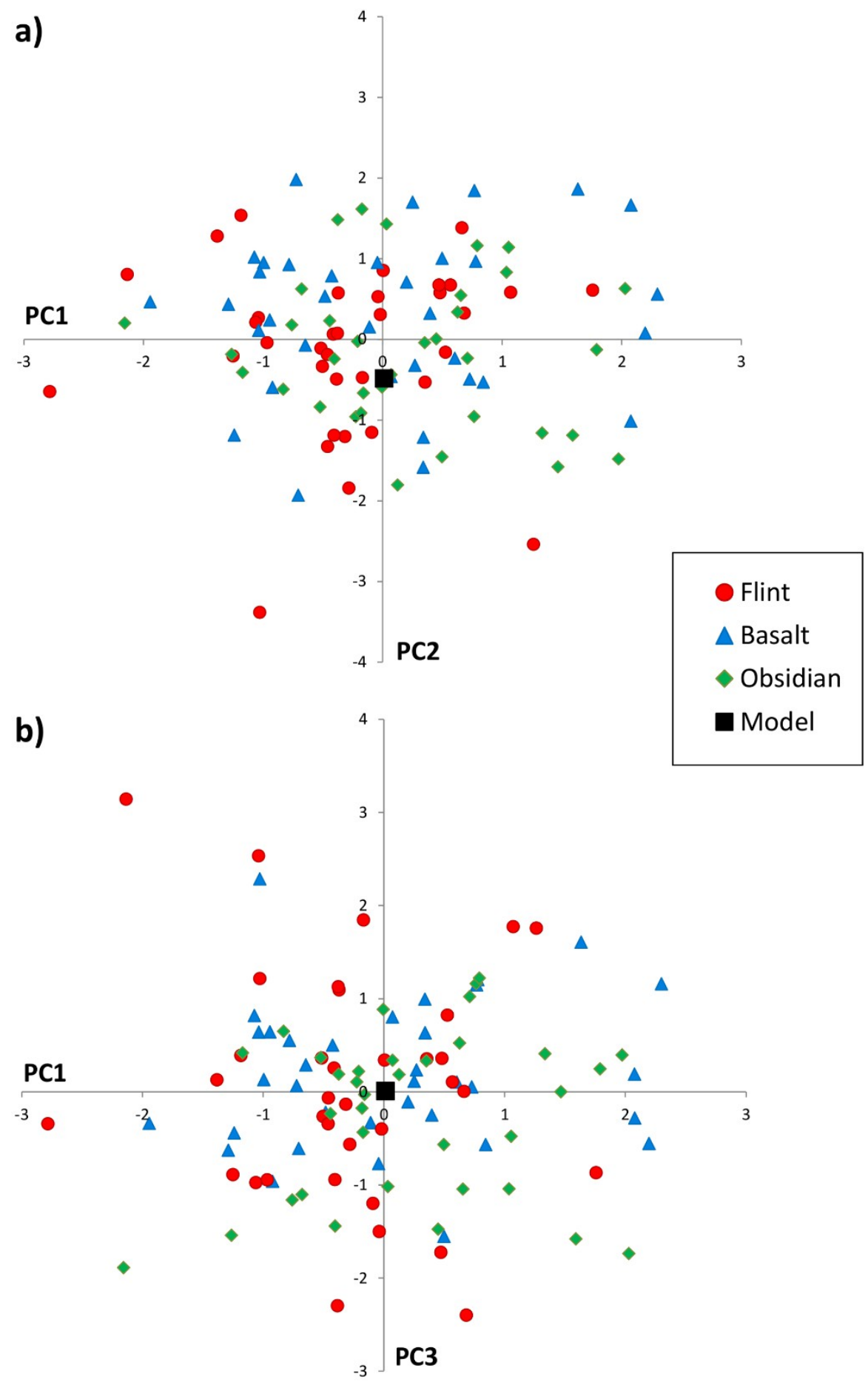

Figure 3: (a) Principal co-ordinates 1 and 2 of morphometric shape data plotted against each other, show variation in the three raw materials compared to the target model handaxe. (b) PC1 and PC3 plotted against each other (from Eren et al. 2014). Respectively, the first three PCs account for $36.4 \%, 12.1 \%$, and $11.0 \%$ of total shape variance. Note the position of the model handaxe, close to the center of the distribution in each of these two plots. 


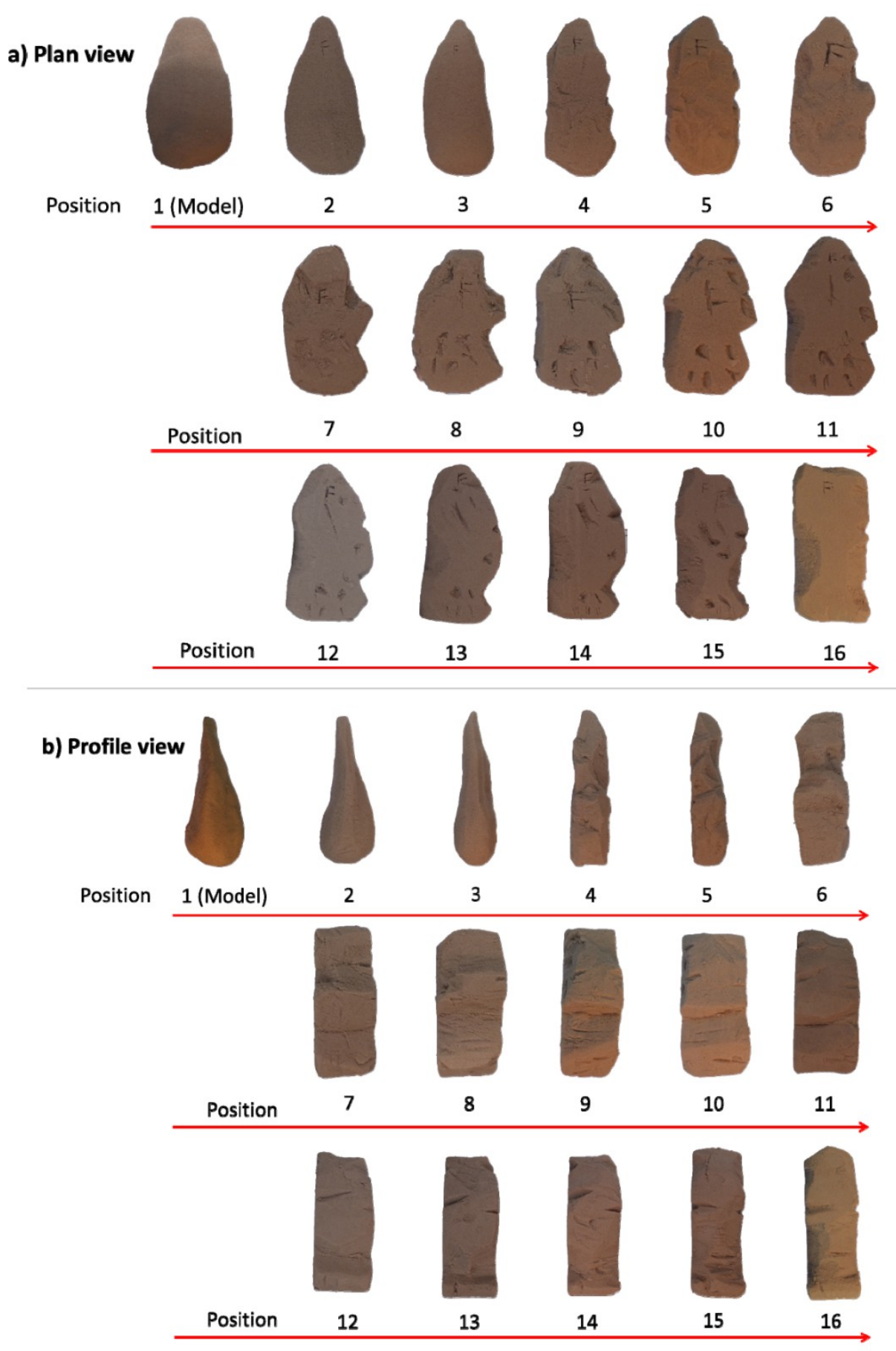

Figure 4: The cumulative effects of copying error. Plan view (a) and profile (b) views of shapes produced by 15 participants copying the shape produced by a previous participant in the form of a "transmission chain" (starting model "handaxe" shown upper left). Each copy in the chain was shaped from standardized, high density foam blocks $(22.3 \times 11 \times 7.8 \mathrm{~cm})$ using a plastic table knife. Despite the fact that the production of these shapes requires no specialized skills or knowledge, disintegration of the initial "handaxe" shape is readily visible over the course of this transmission chain. 


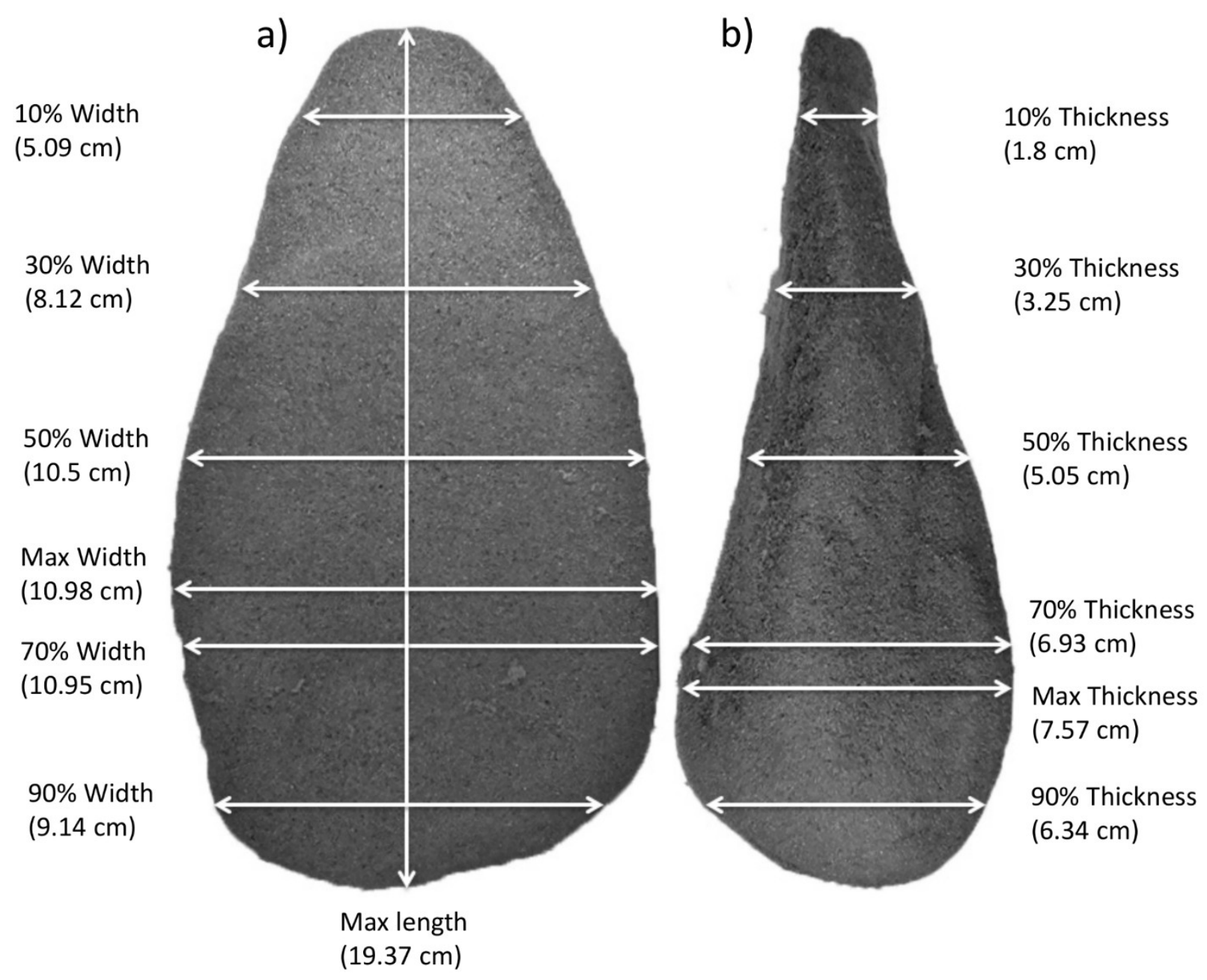

Figure 5: Foam "handaxe" provided to participants to copy in the imitation versus emulation copying experiment (Schillinger et al. 2015). 


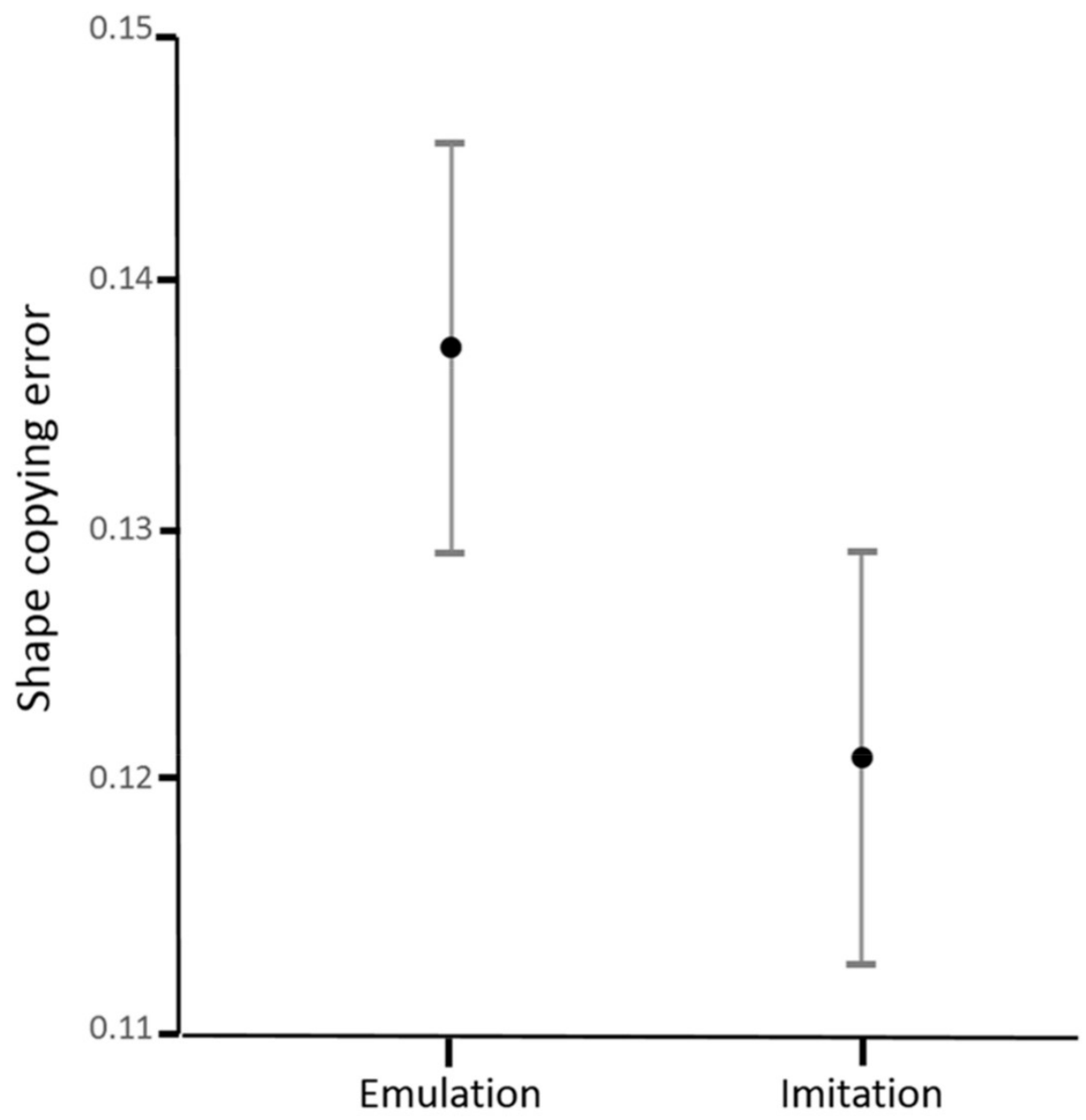

Figure 6: Mean levels of shape copying error in the emulation versus imitation conditions of Schillinger et al. (2015). Whiskers mark in each case mark +/- one standard error. 

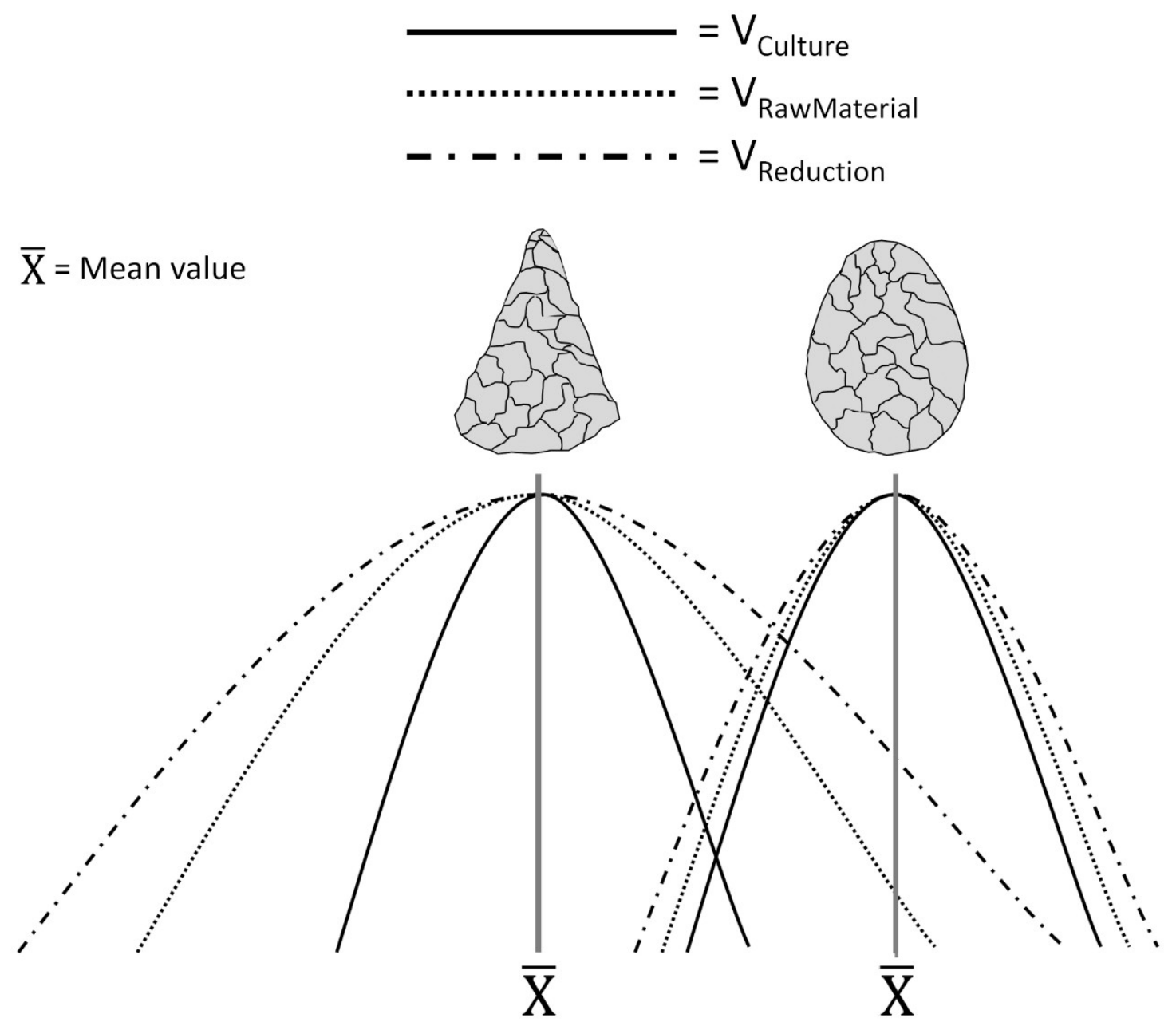

Figure 7: "Quantitative genetic" model of influences on handaxe variation in two different assemblages (see Lycett \& von Cramon-Taubadel 2015). The total variance of a given trait (e.g., handaxe "width") measured in different sets of lithic artifacts may be the result of combined effects of socially learned factors, raw material factors, and differential reduction. Patterns of variation in different sets of artifacts may overlap in total variation, but still exhibit statistically significant differences in mean ( $X^{\prime}$ ) variation. Note that the particular order of the alternative sources of variation here is schematic; in some instances, raw material or culture may be a more prominent sources of within-population variation rather than reduction, for instance. As long as there are heritable (i.e., cultural) behaviors underpinning the expression of a given trait, then, cultural evolutionary factors (selective biases or drift) can lead to exaggerated differences between populations over the course of subsequent transmission events, even in the face of considerable overlap between assemblages due to non-heritable factors. 
Figure 8: While there will inevitably be practical limits on the potential variability that functionally viable "handaxes" can exhibit, there is still potential for variability, which may fluctuate over the course of time and space (see e.g., Isaac, 1972; Gowlett et al., 2001). Our experiments highlight the role of imitation in maintaining general integrity over the course of time in this model, ameliorating the effects of copying error. Drift and/or selection will, however, lead to divergences of pattern, statistically attestable over long-term, short-term, local, and more geographically widespread levels. Principles drawn from quantitative genetics demonstrate how such patterns may occur, even in the face of variability caused by nonheritable factors such as raw material and/or resharpening (see Figure 7). 